ESAIM: M2AN 49 (2015) 141-170

DOI: $10.1051 / \mathrm{m} 2 \mathrm{an} / 2014027$
ESAIM: Mathematical Modelling and Numerical Analysis

www.esaim-m2an.org

\title{
ANALYSIS OF EDDY CURRENT FORMULATIONS IN TWO-DIMENSIONAL DOMAINS WITH CRACKS *
}

\author{
Stephanie Lohrengel ${ }^{1}$ And Serge Nicaise ${ }^{2}$
}

\begin{abstract}
In this paper, the eddy current problem in a two-dimensional conductor containing a crack is studied. The decomposition of the electric field into a piecewise regular part and a singular part deriving from scalar potentials localized at the crack tip and at the crack mouth is proved. At the crack mouth, the electric field is shown to have standard singularities inside the conductor, but presents a singularity outside the conductor that does not belong to the classical $L^{2}$-space. Well-posedness of the $\boldsymbol{E}$-based model and the $\boldsymbol{A}-\psi$-formulation of combined potentials are proved and an un-gauged discretization of the latter formulation is discussed.
\end{abstract}

Mathematics Subject Classification. 35B65, 35Q60, 65N30.

Received November 7, 2013. Revised May 13, 2014.

Published online January 14, 2015.

\section{INTRODUCTION}

The present paper is concerned with the analysis of eddy currents in the presence of cracks.

Eddy current simulations have become an important research area due to numerous applications in electrical engineering. Eddy current testing, for example, as a particular technique of non destructive testing, remains one of the most popular tools in the quality control of conducting test pieces, and an important number of papers in the electric engineering community, mostly based on integral equation techniques, is devoted to numerical methods for crack detection (see e.g. $[11,12,14,15,27]$ ). In a typically configuration of eddy current testing, a coil carrying an alternative current is placed in proximity to the conducting test piece. The coil's magnetic field induces the eddy currents in the conductor which generate measurable variations of the impedance of the coil. In the presence of a crack, the eddy currents are deviated and the electromagnetic response allows for the detection of the defect. Due to the limited penetration depth of the currents, eddy current testing is mostly used to detect cracks that are situated near the surface.

In the mathematical community, eddy current models have gathered much interest in relation with the topological properties of the device. Several formulations for the eddy current problem have been suggested (see

Keywords and phrases. Eddy current problems, domains with cracks, singularities of solutions.

* The authors would like to thank the anonymous referees for useful comments and suggestions that improved the applicability of the present paper.

1 Laboratoire de Mathématiques, EA 4535, FR CNRS 3399, UFR Sciences Exactes et Naturelles, Université de Reims Champagne-Ardenne, Moulin de la Housse - B.P. 1039, 51687 Reims cedex 2, France. stephanie.lohrengel@univ-reims.fr

2 LAMAV, EA 4015, FR CNRS 2956, Université de Valenciennes et du Hainaut Cambrésis, Le Mont Houy, 59313 Valenciennes cedex 9, France. serge.nicaise@univ-valenciennes.fr 
the very complete book of Alonso and Valli [1] and references therein). They differ essentially in the choice of the unknowns, which can be either the electric or the magnetic field, but also combined vector and scalar potentials. The numerical approximation by means of finite element or boundary element methods has been investigated in $[7,8,21,29]$ among others. The asymptotic analysis of the eddy current model as the limit configuration of the full Maxwell equations at low frequencies has been addressed in [3]. For the local behavior of the eddy currents in the vicinity of corners and edges, we refer to [20]. However, these papers always consider domains without cracks, and, to the best of our knowledge, a full mathematical description of the behavior of the fields near cracks is actually not available.

Domains with cracks are widely studied in mechanical engineering and new methods as the XFEM (extended finite element method) have emerged recently to deal advantageously with crack propagation (see e.g. [23,32,37]). In these configurations, standard analysis tools apply to describe the local behavior of the fields near the crack tip and no singularity occurs at the crack mouth since it is in general situated at the boundary of the domain. General crack problems for elliptic systems have been investigated for example in $[18,19]$ and describe the behavior near cracks which arise in the interior of the body.

Eddy current simulations, however, are characterized by the computation of the fields in the conductor as well as in the surrounding insulating medium. Emerging cracks present two different types of singularities: one at the crack tip, the other at the crack mouth where the crack emerges. From a mathematical point of view, the crack mouth is situated at the interface between the conductor and the exterior domain, and the analysis of the fields near the crack mouth is thus not standard.

In the present paper, we give a detailed description of the electric field near a one-dimensional straight crack in a domain of $\mathbb{R}^{2}$ representing the conductor surrounded by an insulating material. We suppose the crack to be emerging at the conductor's surface and emphasize on the behavior of the fields near the crack tip on the one hand and the crack mouth where the crack emerges, on the other. We prove a decomposition theorem that allows to split the electric field into a regular and a singular part. It turns out that the singular part may be derived from scalar potentials which, in the presence of an emerging crack, are localized at the crack tip and at the crack mouth. At the crack tip, the asymptotic analysis is obtained by generalizing well-known decomposition results of the electromagnetic field in the vicinity of geometric singularities [10,17]. At the crack mouth, however, the situation is quite different. Indeed, we prove that the electric field has standard singularities inside the conductor whereas it presents a singularity outside the conductor which actually does not belong to $L^{2}$. Our results are in agreement with the observations made by the physical or electrical engineering community [13].

The paper is organized in the following way: in Section 2 we make precise the eddy current model for the electric field in the presence of a crack and recall the functional spaces that will be used in the sequel. The main result of the paper is presented in Section 3: we introduce an appropriate energy space for the electric field and prove the decomposition of its elements into a regular and a singular part. Section 4 is devoted to the analysis of two variational formulations of the eddy current model. The $\boldsymbol{E}$-formulation, in terms of the electric field, is directly based on a sub-space of the energy space. However, it turns out that this formulation is not well suited for discretization due to the constraints contained in the energy space. We therefore recall the $\boldsymbol{A}$ - $\psi$-formulation which is standard in electric engineering. Here, the electric field is given as the sum of a vector potential $\boldsymbol{A}$ defined in the whole computational domain and the gradient of a scalar potential $\psi$ which is given only in the conductor. We prove that both the $\boldsymbol{E}$ - and the $\boldsymbol{A}$ - $\psi$-formulation are well posed and show that the two formulations are equivalent. With regards to the numerical implementation, we also discuss whether gauging of the vector and scalar potentials is necessary or not and show in Section 5 some numerical results of the simulation of eddy currents in a circular conductor containing a crack.

\section{Setting of the Problem}

\subsection{The eddy current model}

The eddy current problem is derived from the full Maxwell equations by neglecting the displacement field. In a time-harmonic setting, this approximation is admissible at the low frequency range since the product of 
the angular frequency $\omega$ and the electric permittivity $\varepsilon$ is small compared to the conductivity $\sigma$ of the body (see [20] for a discussion of this item and [3] for the asymptotic analysis). At a given angular frequency $\omega>0$, we thus have

$$
\begin{aligned}
i \omega \boldsymbol{B}+\operatorname{curl} \boldsymbol{E} & =0, \\
\operatorname{curl} \boldsymbol{H} & =\boldsymbol{J},
\end{aligned}
$$

where $\boldsymbol{B}$ denotes the magnetic induction, $\boldsymbol{H}$ and $\boldsymbol{E}$ are the magnetic and electric field, and $\boldsymbol{J}=\boldsymbol{J}_{s}+\boldsymbol{J}_{\text {eddy }}$ represents the total current density which splits into the impressed current density $\boldsymbol{J}_{s}$ on the one hand and the eddy currents $\boldsymbol{J}_{\text {eddy }}$ on the other.

The constitutive laws for a linear conducting material read as follows

$$
\left\{\begin{array}{l}
\boldsymbol{B}=\mu \boldsymbol{H}, \\
\boldsymbol{J}_{\text {eddy }}=\sigma \boldsymbol{E},
\end{array}\right.
$$

where $\mu$ and $\sigma$ denote respectively the magnetic permeability and the conductivity of the material. Now, consider a homogeneous and isotropic conductor $Q_{c}$ with permeability $\mu_{c}>0$ and conductivity $\sigma_{c}>0$. We assume $Q_{c}$ to be connected, lipschitz and piecewise regular. Further, let $Q$ be a bounded, simply connected domain with connected boundary $\Gamma_{\infty}=\partial Q$ such that $\overline{Q_{c}} \subset Q$ and denote by $Q_{e}=Q \backslash \bar{Q}_{c}$ the exterior domain. For the sake of simplicity, we also assume that $Q$ is regular. Let $\Gamma_{0}=\partial Q_{c}$ be the interface between the exterior domain $Q_{e}$ and the conductor $Q_{c}$. Let $Q_{e}$ be filled by an insulating material like air with permeability $\mu_{e}>0$ and zero conductivity. Hence, the functions $\mu$ and $\sigma$ are piecewise constant and satisfy

$$
\mu=\left\{\begin{array}{ll}
\mu_{c} & \text { in } Q_{c}, \\
\mu_{e} & \text { in } Q_{e},
\end{array} \text { and } \sigma=\left\{\begin{array}{lll}
\sigma_{c} & \text { in } Q_{c} \\
0 & \text { in } & Q_{e}
\end{array}\right.\right.
$$

We thus see from (2.2) that the eddy currents are supported only by the conductor.

We make the following assumptions on $\boldsymbol{J}_{s}$,

$$
\operatorname{div} \boldsymbol{J}_{s}=0 \text { in } Q \text { and } \operatorname{supp}\left(\boldsymbol{J}_{s}\right) \subset Q_{e} .
$$

This models for instance the case of a coil excitation outside the conductor and fits with our concerns (see [8] for a discussion of other types of excitation leading to skin effect problems).

Taking into account the constitutive laws and eliminating the magnetic field, we deduce from (2.1) that, in the absence of a crack, the electric field $\boldsymbol{E}$ is solution of the following problem

$$
\begin{cases}\operatorname{curl} \mu^{-1} \operatorname{curl} \boldsymbol{E}+i \omega \sigma \boldsymbol{E}=-i \omega \boldsymbol{J}_{s} & \text { in } \quad Q \\ \boldsymbol{E} \times \boldsymbol{n}=0 & \text { on } \Gamma_{\infty} .\end{cases}
$$

(2.5) has to be completed by the following gauge conditions on the exterior field $\boldsymbol{E}_{e}$ which are obtained from the full Maxwell equations before passing to the limit (see again $[3,20]$ ).

$$
\left\{\begin{array}{l}
\operatorname{div} \boldsymbol{E}_{e}=0 \quad \text { in } Q_{e}, \\
\left\langle\boldsymbol{E}_{e} \cdot \boldsymbol{n}, 1\right\rangle=0 \text { on any connected component of } \Gamma_{0} .
\end{array}\right.
$$

From now on, we restrict ourselves to a two-dimensional configuration with a conductor that presents a one-dimensional straight crack emerging at some point $\boldsymbol{x}_{0}$ of $\Gamma_{0}$. Without loss of generality, we assume that the interface $\Gamma_{0}$ between the conductor and the surrounding medium has one single component. This does not affect the analysis near the crack and simplifies the presentation. The crack tip $\boldsymbol{x}^{*}$ belongs to the interior of the conductor and the crack is thus the (closed) segment

$$
\Sigma=\left\{s \boldsymbol{x}^{*}+(1-s) \boldsymbol{x}_{0} \mid s \in[0,1]\right\} .
$$



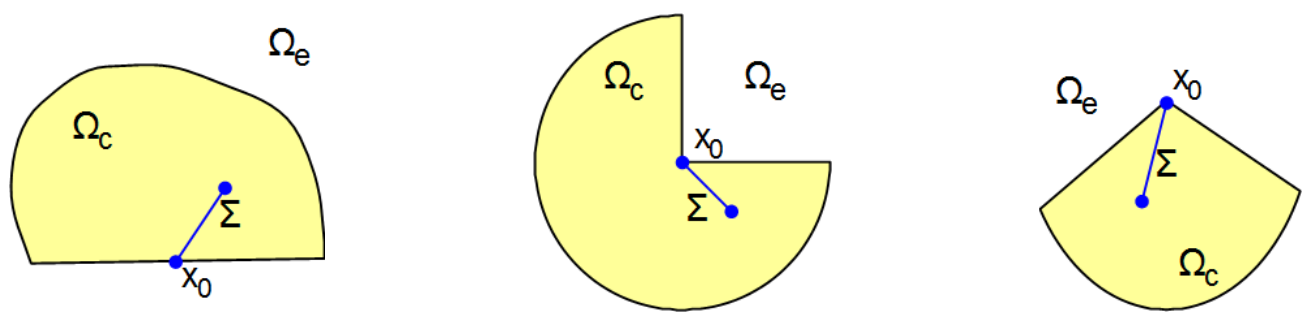

FiguRE 1. Examples of eddy-current devices.

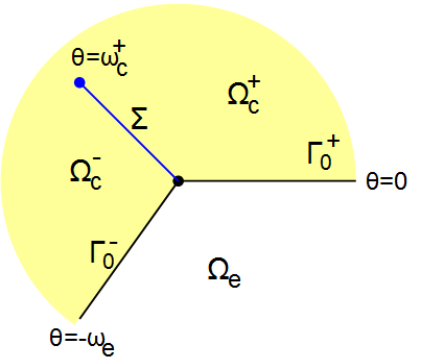

FIGURE 2. Local polar coordinates near the crack mouth.

For convenience, we have assumed that the crack is straight. In order to simplify the presentation, we further assume that the conductor $Q_{c}$ has at most one angular point which in addition coincides with the crack mouth $\boldsymbol{x}_{0}$ (see Fig. 1). Indeed, the behavior of the electric field in a domain with vertices away from $\boldsymbol{x}_{0}$ can be deduced from the results in [20] where eddy current formulations on general polyhedral conductors have been studied. The results stated below should carry over to a configuration with a curved crack or a curvilinear interface $\Gamma_{0}$.

Now, let $\Omega_{c}=Q_{c} \backslash \Sigma$ denote the open set representing the conductor outside the crack and set $\Omega=Q \backslash \Sigma$. Notice that the exterior domain $\Omega_{e}=\Omega \backslash \bar{\Omega}_{c}$ coincides with $Q_{e}$. We define the unit tangent vector of the crack by $\tau_{\Sigma}=\overrightarrow{\boldsymbol{x}_{0} \boldsymbol{x}^{*}} /\left\|\overrightarrow{\boldsymbol{x}_{0} \boldsymbol{x}^{*}}\right\|$, and we denote by $\boldsymbol{n}_{\Sigma}$ the unit normal vector on $\Sigma$ such that $\left(\boldsymbol{n}_{\Sigma}, \tau_{\Sigma}\right)$ forms a direct system. In order to distinguish the two sides of the conductor near the crack, we introduce the sets $\Omega_{c}^{+}=\left\{\boldsymbol{x} \in \Omega_{c} \mid \boldsymbol{x} \cdot \boldsymbol{n}_{\Sigma}>0\right\}$ and $\Omega_{c}^{-}=\left\{\boldsymbol{x} \in \Omega_{c} \mid \boldsymbol{x} \cdot \boldsymbol{n}_{\Sigma}<0\right\}$. The interior angle $\omega_{c}$ at $\boldsymbol{x}_{0}$ is split by the crack into two angles $\omega_{c}^{+}$and $\omega_{c}^{-}$such that $\omega_{c}^{+}+\omega_{c}^{-}=\omega_{c}$. The exterior angle at $\boldsymbol{x}_{0}$ is denoted by $\omega_{e}$. Finally, let $\Gamma_{0}^{ \pm}$be the part of $\Gamma_{0}$ in $\Omega_{c}^{ \pm}$and define $\Gamma_{0}^{0}$ as the part of $\Gamma_{0}$ outside the crack mouth, $\Gamma_{0}^{0}=\Gamma_{0} \backslash\left\{\boldsymbol{x}_{0}\right\}$. For any distribution $f \in \mathcal{D}^{\prime}(\Omega)$, we denote $f_{c}$ (resp. $f_{e}$ ) the restriction of $f$ to $\Omega_{c}$ (resp. $\Omega_{e}$ ).

As we shall be interested in the local behavior of vector fields near the crack tip $\boldsymbol{x}^{*}$, we define local polar coordinates $\left(r^{*}, \theta^{*}\right)$ with respect to $\boldsymbol{x}^{*}$, where the two sides of the crack correspond respectively to $\theta^{*}=+\pi$ and $\theta^{*}=-\pi$. We fix a regular cut-off function $\eta^{*}=\eta^{*}\left(r^{*}\right)$, depending only on the distance $r^{*}$, such that $\eta^{*} \equiv 1$ near $\boldsymbol{x}^{*}$ and $\operatorname{supp}\left(\eta^{*}\right) \subset Q_{c}$.

Similarly, we introduce local polar coordinates $\left(r_{0}, \theta_{0}\right)$ near the crack mouth $\boldsymbol{x}_{0}$ where $r_{0}=\left\|\boldsymbol{x}-\boldsymbol{x}_{0}\right\|$ denotes the distance of $\boldsymbol{x} \in \Omega$ to $\boldsymbol{x}_{0}$ and $\theta_{0}=0$ coincides with $\Gamma_{0}^{+}$near $\boldsymbol{x}_{0}$. Then $\theta_{0}=\omega_{c}^{+}$corresponds to the crack $\Sigma$ and $\Gamma_{0}^{-}$is situated locally on the half-line $\theta_{0}=-\omega_{e}$ (see Fig. 2). We recall that $\Omega_{e}$ coincides with an open sector of angle $\omega_{e}$ in a neighborhood $\mathcal{V}\left(\boldsymbol{x}_{0}\right)$ of $\boldsymbol{x}_{0}: \Omega_{e} \cap \mathcal{V}\left(\boldsymbol{x}_{0}\right)=\left\{\left(r_{0} \cos \theta_{0}, r_{0} \sin \theta_{0}\right) \mid 0<r_{0}<R,-\omega_{e}<\theta_{0}<0\right\}$.

We thus choose a second cut-off function $\eta_{0}=\eta_{0}\left(r_{0}\right)$ with respect to $\boldsymbol{x}_{0}$ such that $\operatorname{supp}\left(\eta_{0}\right) \subset \mathcal{V}\left(\boldsymbol{x}_{0}\right)$. We further assume that the supports of $\eta^{*}$ and $\eta_{0}$ do not intersect. 
Notice that in a two-dimensional setting, we distinguish between the vector curl operator acting on scalar functions and the scalar curl operator acting on vector fields,

$$
\operatorname{curl} \varphi=\left(\begin{array}{c}
\partial_{2} \varphi \\
-\partial_{1} \varphi
\end{array}\right), \operatorname{curl} \boldsymbol{v}=\partial_{1} v_{2}-\partial_{2} v_{1}
$$

Similarly, the cross product of two vectors $\boldsymbol{u}=\left(u_{1}, u_{2}\right)^{t}$ and $\boldsymbol{n}=\left(n_{1}, n_{2}\right)^{t}$ of $\mathbb{R}^{2}$ can be defined by the scalar $\boldsymbol{u} \times \boldsymbol{n}=u_{1} n_{2}-u_{2} n_{1}$.

Here, we are interested in the transverse electric setting: the electric field is invariant in one direction and can be described by the two components $\boldsymbol{E}(\boldsymbol{x})=\left(E_{1}\left(x_{1}, x_{2}\right), E_{2}\left(x_{1}, x_{2}\right)\right)^{t}$.

According to [11], the tangential component of the electric field $\boldsymbol{E}$ is discontinuous across the crack and curl $\boldsymbol{E}$ does not, in general, belong to $L^{2}(Q)$ but only to $L^{2}(\Omega)$. By definition, an ideal crack does not allow any current to pass across it. This yields the boundary condition $\boldsymbol{J} \cdot \boldsymbol{n}_{\Sigma}=0$ on $\Sigma$ for the total current $\boldsymbol{J}=\sigma \boldsymbol{E}+\boldsymbol{J}_{s}$. Under the assumption (2.4), we thus get the boundary condition

$$
\boldsymbol{E} \cdot \boldsymbol{n}_{\Sigma}=0 \text { on } \Sigma
$$

for the electric field. The eddy-current problem in a cracked domain finally reads

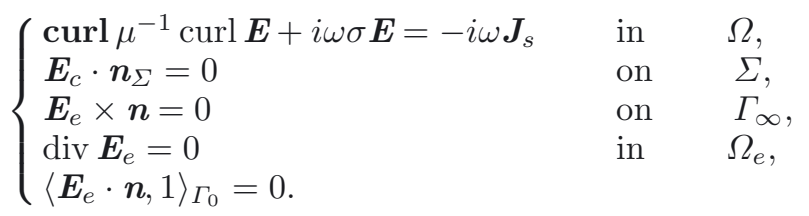

Moreover, notice that the first equation in (2.8) yields

$$
\operatorname{div}(\sigma \boldsymbol{E})=0 \text { in } \Omega,
$$

which implies the continuity of the normal trace of the field $\sigma \boldsymbol{E}$ across the interface $\Gamma_{0}$ outside the crack mouth. Since $\sigma_{e}=0$ in $\Omega_{e}$, we simply get

$$
\boldsymbol{E}_{c} \cdot \boldsymbol{n}=0 \text { on } \Gamma_{0}^{0} .
$$

Since $\boldsymbol{E}_{c} \cdot \boldsymbol{n}$ also vanishes on $\Sigma$, we finally have

$$
\boldsymbol{E}_{c} \cdot \boldsymbol{n}=0 \text { on } \partial \Omega_{c} .
$$

\subsection{Functional framework}

In this subsection, we fix the notations and recall the definition of some useful vector spaces. For an open subset $\mathcal{O}$ of $\mathbb{R}^{n}$ and $m \in \mathbb{N}$, we denote by $H^{m}(\mathcal{O})$ the usual Sobolev space of functions defined on $\mathcal{O}$ with square integrable derivatives up to order $m$. On $H^{m}(\mathcal{O}),\|\cdot\|_{m, \mathcal{O}}$ denotes the standard norm. The following definition of fractional order Sobolev spaces can be found for example in [26]. Let $s\rangle 0$ such that $s=m+\sigma$ with $m \in \mathbb{N}$ and $0<\sigma<1$. We define

$$
H^{s}(\mathcal{O})=\left\{f \in H^{m}(\mathcal{O})\left|\int_{\mathcal{O}} \int_{\mathcal{O}} \frac{\left|D^{\alpha} f(\boldsymbol{x})-D^{\alpha} f(\boldsymbol{y})\right|^{2}}{|\boldsymbol{x}-\boldsymbol{y}|^{n+2 \sigma}} \mathrm{d} \boldsymbol{x} \mathrm{d} \boldsymbol{y}<+\infty, \forall \alpha=\left(\alpha_{1}, \ldots, \alpha_{n}\right):\right| \alpha \mid=m\right\} .
$$

On $H^{s}(\mathcal{O})$, the canonical norm is given by

$$
\|f\|_{s, \mathcal{O}}=\left(\|f\|_{m, \mathcal{O}}^{2}+\sum_{|\alpha|=m} \int_{\mathcal{O}} \int_{\mathcal{O}} \frac{\left|D^{\alpha} f(\boldsymbol{x})-D^{\alpha} f(\boldsymbol{y})\right|^{2}}{|\boldsymbol{x}-\boldsymbol{y}|^{n+2 \sigma}} \mathrm{d} \boldsymbol{x} \mathrm{d} \boldsymbol{y}\right)^{1 / 2} .
$$

For $s<0$, the space $H^{-s}(\mathcal{O})$ is the dual space of $H_{0}^{s}(\mathcal{O})$, the closure of $\mathcal{D}(\mathcal{O})$ with respect to the norm $\|\cdot\|_{s, \mathcal{O}}$. 
Special attention has to be paid if $s=1 / 2$. We denote by $\widetilde{H}^{1 / 2}(\mathcal{O})$ the space of functions $f$ defined on $\mathcal{O}$ such that the extension of $f$ by 0 outside $\mathcal{O}$ belongs to $H^{1 / 2}\left(\mathbb{R}^{n}\right)$. The following application defines a norm on $\widetilde{H}^{1 / 2}(\mathcal{O})$ if $\mathcal{O}$ is a Lipschitz domain,

$$
\|f\|_{\sim, 1 / 2, \mathcal{O}}=\left(\|f\|_{1 / 2, \mathcal{O}}^{2}+\int_{\mathcal{O}} \frac{|f(\boldsymbol{x})|^{2}}{\rho(\boldsymbol{x})} \mathrm{d} \boldsymbol{x}\right)^{1 / 2} .
$$

Here, $\rho(\boldsymbol{x})$ denotes the distance from $\boldsymbol{x}$ to the boundary of $\mathcal{O}$. Following the notations in $[26], \widetilde{H}^{-1 / 2}(\mathcal{O})$ will denote the dual space of $\widetilde{H}^{1 / 2}(\mathcal{O})$ which differs from $H^{-1 / 2}(\mathcal{O})$ (see [26] for a discussion of this item). Notice that this definition is equivalent to the definition of the space $H_{00}^{-1 / 2}(\mathcal{O})$ in [16].

Now, let $\mathcal{O}^{+}$and $\mathcal{O}^{-}$be two open segments in $\mathbb{R}^{2}$ such that $\mathcal{O}^{+} \cap \mathcal{O}^{-}=\emptyset$ and $\overline{\mathcal{O}^{+}} \cap \overline{\mathcal{O}^{-}}=\{S\}$ where $S$ is a point of $\mathbb{R}^{2}$. For $s>0$ small enough, let $\boldsymbol{x}(s)$ (resp. $\boldsymbol{x}(-s)$ ) be the point on $\mathcal{O}^{+}$(resp. $\mathcal{O}^{-}$) whose distance to $S$ is $s$. Then, for elements $f^{ \pm} \in H^{1 / 2}\left(\mathcal{O}^{ \pm}\right)$we write $f^{+} \equiv f^{-}$at $S$ if

$$
\int_{0}^{\delta} \frac{\left|f^{+}(\boldsymbol{x}(+s))-f^{-}(\boldsymbol{x}(-s))\right|^{2}}{s} \mathrm{~d} s<+\infty .
$$

If $f^{+}$and $f^{-}$are Hölder-continuous near $S, f^{+} \equiv f^{-}$is equivalent to $f^{+}(S)=f^{-}(S)$. Obviously, any function $f$ in $\widetilde{H}^{1 / 2}(\mathcal{O})$ satisfies $f \equiv 0$ at the extremities of $\mathcal{O}$ if $\mathcal{O}$ is an open segment of $\mathbb{R}^{2}$.

In this paper, we are concerned with domains containing cracks. In particular, such domains are not of Lipschitz type. However, the definitions here above can be adapted and most of the usual properties of Sobolev spaces (excepted the extension property) carry over to a cracked domain.

In the sequel, bold letters indicate spaces of vector fields, e.g. $\boldsymbol{H}^{1}(\mathcal{O})=\left(H^{1}(\mathcal{O})\right)^{n}$. The following vector spaces are involved in the mathematical formulation of the Maxwell equations for $\mathcal{O} \subset \mathbb{R}^{2}$.

$$
\begin{aligned}
\mathcal{H}(\operatorname{curl} ; \mathcal{O}) & =\left\{\boldsymbol{v} \in \mathbf{L}^{2}(\mathcal{O}) \mid \operatorname{curl} \boldsymbol{v} \in L^{2}(\mathcal{O})\right\}, \\
\mathcal{H}(\operatorname{div} ; \mathcal{O}) & =\left\{\boldsymbol{v} \in \mathbf{L}^{2}(\mathcal{O}) \mid \operatorname{div} \boldsymbol{v} \in L^{2}(\mathcal{O})\right\}
\end{aligned}
$$

For any field $\boldsymbol{v} \in \mathcal{H}(\operatorname{curl} ; \mathcal{O})$, we can define its tangential trace $\gamma_{t} \boldsymbol{v} \in H^{-1 / 2}(\partial \mathcal{O})$ on the boundary $\partial \mathcal{O}$ as the continuous extension of the application $\boldsymbol{v} \mapsto(\boldsymbol{v} \times \boldsymbol{n})_{\mid \partial \mathcal{O}}$ which is well defined for regular fields. In the same way, the normal trace $\gamma_{n} \boldsymbol{v}$ is defined as the extension of the application $\boldsymbol{v} \mapsto(\boldsymbol{v} \cdot \boldsymbol{n})_{\mid \partial \mathcal{O}}$ and belongs to $H^{-1 / 2}(\partial \mathcal{O})$ for any field $\boldsymbol{v} \in \mathcal{H}(\operatorname{div} ; \mathcal{O})$.

This allows the definition of the following spaces

$$
\begin{aligned}
\mathcal{H}_{0}(\operatorname{curl} ; \mathcal{O}) & =\left\{\boldsymbol{v} \in \mathcal{H}(\operatorname{curl} ; \mathcal{O}) \mid \gamma_{t} \boldsymbol{v}=0 \text { on } \partial \mathcal{O}\right\} \\
\mathcal{H}_{0}(\operatorname{div} ; \mathcal{O}) & =\left\{\boldsymbol{v} \in \mathcal{H}(\operatorname{div} ; \mathcal{O}) \mid \gamma_{n} \boldsymbol{v}=0 \text { on } \partial \mathcal{O}\right\}
\end{aligned}
$$

If $D \subset \partial \mathcal{O}$, we denote by $\mathcal{H}_{0, D}(\operatorname{curl} ; \mathcal{O})$ the subspace of $\mathcal{H}(\operatorname{curl} ; \mathcal{O})$ of fields $\boldsymbol{v}$ such that $\gamma_{t} \boldsymbol{v}=0$ on $D$. In the same way, we define $\mathcal{H}_{0, D}(\operatorname{div} ; \mathcal{O})$ and $H_{0, D}^{1}(\mathcal{O})$. Notice that for any $\boldsymbol{v} \in \mathcal{H}(\operatorname{curl} ; \mathcal{O})$, the tangential trace $\left(\gamma_{t} \boldsymbol{v}\right)_{\mid D}$ on a closed curve $D$ is defined as an element in $H^{-1 / 2}(D)$. However, if $D$ has a boundary, $\left(\gamma_{t} \boldsymbol{v}\right)_{\mid D}$ only belongs to $\widetilde{H}^{-1 / 2}(D)$.

\section{A DECOMPOSITION THEOREM}

Assume for a moment that the solution of the eddy current problem is regular near the crack mouth $\boldsymbol{x}_{0}$. The boundary condition $\boldsymbol{E}_{c} \cdot \boldsymbol{n}=0$ on $\Sigma$ and $\Gamma_{0}^{ \pm}$thus implies that $\boldsymbol{E}_{c}\left(\boldsymbol{x}_{0}\right)=\mathbf{0}$ since $\boldsymbol{E}_{c}$ is orthogonal at $\boldsymbol{x}_{0}$ to the two linear independent vectors $\boldsymbol{n}_{\Sigma}$ and $\boldsymbol{n}_{\Gamma_{0}^{+}}$(resp. $\boldsymbol{n}_{\Sigma}$ and $\boldsymbol{n}_{\Gamma_{0}^{-}}$if $\omega_{c}^{+}=\pi$ ). If $\boldsymbol{E}$ belongs to $\mathcal{H}(\operatorname{curl} ; \Omega)$, the tangential trace of $\boldsymbol{E}$ across $\Gamma_{0}$ is continuous away from $\boldsymbol{x}_{0}$. Hence, $\left(\boldsymbol{E}_{\mathrm{e}}^{+} \times \boldsymbol{n}\right)\left(\boldsymbol{x}_{0}\right)=\left(\boldsymbol{E}_{c}^{+} \times \boldsymbol{n}\right)\left(\boldsymbol{x}_{0}\right)=0$ and, in 
the same way, $\left(\boldsymbol{E}_{\mathrm{e}}^{-} \times \boldsymbol{n}\right)\left(\boldsymbol{x}_{0}\right)=0$. In other words, both the interior and exterior part of the electric field vanish at the crack mouth. This is not conforming with the analysis in [11] which exhibits a singularity arising from the potential drop across the crack mouth.

We therefore introduce the following weighted Sobolev spaces defined on $\Omega_{e}$ depending on a weight parameter $\alpha>0$ :

$$
L_{\alpha}^{2}\left(\Omega_{e}\right)=\left\{f \in \mathcal{D}^{\prime}\left(\Omega_{e}\right) \mid r_{0}^{\alpha} f \in L^{2}\left(\Omega_{e}\right)\right\}
$$

and

$$
H^{1, \alpha}\left(\Omega_{e}\right)=\left\{f \in \mathcal{D}^{\prime}\left(\Omega_{e}\right) \mid r_{0}^{\alpha-1} f \in L^{2}\left(\Omega_{e}\right), r_{0}^{\alpha} \partial_{i} f \in L^{2}\left(\Omega_{e}\right) \forall i=1,2\right\} .
$$

Lemma 3.1. For any $p$ such that $1 \leq p<\frac{2}{\alpha+1}$, the weighted space $L_{\alpha}^{2}\left(\Omega_{e}\right)$ is embedded continuously in $L^{p}\left(\Omega_{e}\right)$.

Proof. Let $u \in L_{\alpha}^{2}\left(\Omega_{e}\right)$. Hence $u$ may be factorized into $u=r_{0}^{-\alpha} r_{0}^{\alpha} u$ where $r_{0}^{\alpha} u$ belongs to $L^{2}\left(\Omega_{e}\right)$. Now, $r_{0}^{-\alpha}$ belongs to $L^{q}\left(\Omega_{e}\right)$ whenever $-\alpha q+2>0$ which yields $\frac{1}{q}>\frac{\alpha}{2}$. Thus, $u^{p}=r_{0}^{-\alpha p}\left(r_{0}^{\alpha} u\right)^{p}$ belongs to $L^{1}\left(\Omega_{e}\right)$ if $\frac{p}{q}+\frac{p}{2}=1$ since $r_{0}^{-\alpha p} \in L^{q / p}\left(\Omega_{e}\right)$ and $\left(r_{0}^{\alpha} u\right)^{p} \in L^{2 / p}\left(\Omega_{e}\right)$. This yields

$$
\frac{1}{p}=\frac{1}{q}+\frac{1}{2}>\frac{\alpha+1}{2}
$$

and

$$
\|u\|_{L^{p}\left(\Omega_{e}\right)}^{p} \leq\left\|r_{0}^{-\alpha}\right\|_{L^{q}\left(\Omega_{e}\right)}^{p}\left\|r_{0}^{\alpha} u\right\|_{L^{2}\left(\Omega_{e}\right)}^{p}
$$

The following proposition describes the behavior of the solution of a scalar boundary value problem in $\Omega_{e}$ with piecewise regular boundary data which are allowed to be discontinuous at $\boldsymbol{x}_{0}$.

Proposition 3.2. Let $f \in L^{2}\left(\Omega_{e}\right), g \in H^{1}\left(\Gamma_{0}^{0}\right)$ and $b \in \mathbb{C}$. We define the function $S_{0}$ in the infinite sector of angle $\omega_{e}$ by

$$
S_{0}\left(r_{0}, \theta_{0}\right)=\frac{\theta_{0}}{\omega_{e}}
$$

in local polar coordinates $\left(r_{0}, \theta_{0}\right)$ with respect to $\boldsymbol{x}_{0}$. Let $c_{0}=\left[g\left(\boldsymbol{x}_{0}\right)\right] \in \mathbb{C}$ where the jump $\left[g\left(\boldsymbol{x}_{0}\right)\right]$ is defined by $\left[g\left(\boldsymbol{x}_{0}\right)\right]=g^{+}\left(\boldsymbol{x}_{0}\right)-g^{-}\left(\boldsymbol{x}_{0}\right)$.

There is a unique function $\varphi_{r} \in H^{1}\left(\Omega_{e}\right)$ such that the function

$$
\varphi=\varphi_{r}+c_{0} \eta_{0} S_{0}
$$

is solution of the problem

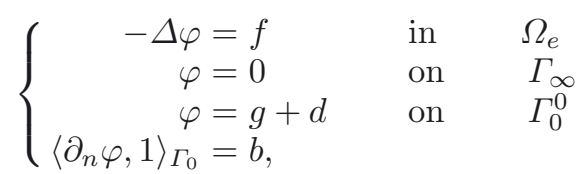

for some $d \in \mathbb{C}$. Moreover, $\varphi_{r}$ and $c_{0}$ satisfy the estimate

$$
\left\|\varphi_{r}\right\|_{1, \Omega_{e}}+\left|c_{0}\right| \lesssim\|f\|_{0, \Omega_{e}}+\left\|\partial_{s} g\right\|_{0, \Gamma_{0}^{0}}+|b| .
$$

Here and below, we write $a \lesssim b$ whenever there is a constant $C>0$ independent from the quantities $a$ and $b$ such that $a \leq C b$.

Notice that $\varphi$ satisfies the Dirichlet boundary condition $\varphi=g$ on $\Gamma_{0}^{0}$ up to some additive constant. More precisely, following [20] (Lem. 2.1), $\varphi$ is the superposition of the solution of a standard Dirichlet boundary problem with data $f$ and $g$ and the solution of $\Delta q=0$ in $\Omega_{e}, q=0$ on $\Gamma_{\infty}, q=$ const. on $\Gamma_{0}$ and $\left\langle\partial_{n} q, 1\right\rangle_{\Gamma_{0}}=1$. This allows to get the estimate (3.5) where the Dirichlet datum $g$ only appears through its derivative. 
Proof. Since $g$ belongs to $H^{1}\left(\Gamma_{0}^{0}\right) \hookrightarrow \mathcal{C}^{0}\left(\Gamma_{0}^{0}\right)$, the restrictions $g^{ \pm}=g_{\mid \Gamma_{0}^{ \pm}}$are continuous at $\boldsymbol{x}_{0}$, and we can define the jump of $g$ at $\boldsymbol{x}_{0},\left[g\left(x_{0}\right)\right]=g^{+}\left(\boldsymbol{x}_{0}\right)-g^{-}\left(\boldsymbol{x}_{0}\right)$.

Then, define the function $\tilde{g}$ by $\tilde{g}=g-\left[g\left(x_{0}\right)\right]\left(\eta_{0} S_{0}\right)_{\mid \Gamma_{0}^{0}}$. We have

$$
\left\{\begin{array}{rll}
\Delta S_{0}=0 & \text { in } & \mathcal{S}_{-\omega_{e}} \\
S_{0}=0 & \text { on } & \left\{\theta_{0}=0\right\}, \\
S_{0}=-1 & \text { on } & \left\{\theta_{0}=-\omega_{e}\right\}
\end{array}\right.
$$

where

$$
\mathcal{S}_{-\omega_{e}}=\left\{\left(r_{0} \cos \theta_{0}, r_{0} \sin \theta_{0}\right) \mid r_{0}>0,-\omega_{e}<\theta_{0}<0\right\}
$$

denotes the infinite sector of angle $\omega_{e}$ between the half-lines $\left\{\theta_{0}=-\omega_{e}\right\}$ and $\left\{\theta_{0}=0\right\}$.

The function $\tilde{g}$ belongs to $H^{1}\left(\Gamma_{0}^{0}\right)$ and we have $\left[\tilde{g}\left(\boldsymbol{x}_{0}\right)\right]=0$ by construction. Therefore, $\tilde{g}$ belongs to $H^{1 / 2}\left(\Gamma_{0}\right) \cap$ $H^{1}\left(\Gamma_{0}^{0}\right)$.

From [20] (Lem. 2.1) we know that there is a unique function $\varphi_{r} \in H^{1}\left(\Omega_{e}\right)$, solution of the problem

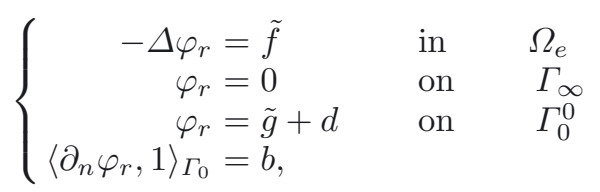

where $\tilde{f}=f+\left[g\left(\boldsymbol{x}_{0}\right)\right] \Delta\left(\eta_{0} S_{0}\right) \in L^{2}\left(\Omega_{e}\right)$ and $d$ is some complex number. Moreover, $\varphi_{r}$ satisfies the estimate

$$
\left\|\varphi_{r}\right\|_{1, \Omega_{e}} \lesssim\|\tilde{f}\|_{0, \Omega_{e}}+\|\tilde{g}\|_{H^{1 / 2}\left(\Gamma_{0}\right) / \mathbb{C}}+|b| .
$$

Then, define $\varphi$ by $\varphi=\varphi_{r}+c_{0} \eta_{0} S_{0}$ with $c_{0}=\left[g\left(\boldsymbol{x}_{0}\right)\right]$. Since $\eta_{0} S_{0}$ belongs to $H^{1, \alpha}\left(\Omega_{e}\right)$ according to Proposition A.1, so does $\varphi$. By construction, we have

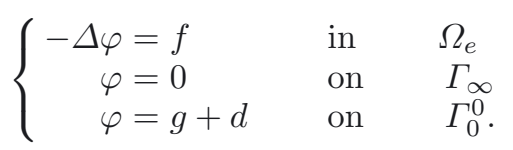

In order to prove that $\varphi$ satisfies the last property in (3.4), we have to evaluate the quantity $\left\langle\partial_{n}\left(\eta_{0} S_{0}\right), 1\right\rangle_{\Gamma_{0}}$. To this end, notice that for any $p$ such that $1<p<2, \partial_{n}\left(\eta_{0} S_{0}\right)$ is an element of $W^{-1 / p, p}\left(\Gamma_{0}\right)$, the dual of $W^{1-1 / p^{\prime}, p^{\prime}}\left(\Gamma_{0}\right)$ with $\frac{1}{p}+\frac{1}{p^{\prime}}=1$, since $\nabla\left(\eta_{0} S_{0}\right) \in \boldsymbol{L}^{p}\left(\Omega_{e}\right)$ and $\Delta\left(\eta_{0} S_{0}\right) \in L^{p}\left(\Omega_{e}\right)$. Consequently, $\left\langle\partial_{n}\left(\eta_{0} S_{0}\right), 1\right\rangle_{\Gamma_{0}}$ is well defined as a duality pairing between $W^{-1 / p, p}\left(\Gamma_{0}\right)$ and $W^{1-1 / p^{\prime}, p^{\prime}}\left(\Gamma_{0}\right)$ and the following Green formula holds

$$
\int_{\Omega_{e}} \Delta\left(\eta_{0} S_{0}\right) \mathrm{d} \boldsymbol{x}=\left\langle\partial_{n}\left(\eta_{0} S_{0}\right), 1\right\rangle_{\Gamma_{0}} .
$$

Since $\Delta\left(\eta_{0} S_{0}\right)=0$ in a neighborhood of $\boldsymbol{x}_{0}$, the previous identity may be written

$$
\left\langle\partial_{n}\left(\eta_{0} S_{0}\right), 1\right\rangle_{\Gamma_{0}}=\lim _{\delta \rightarrow 0} \int_{\Omega_{e} \backslash B\left(\boldsymbol{x}_{0}, \delta\right)} \Delta\left(\eta_{0} S_{0}\right) \mathrm{d} \boldsymbol{x} .
$$

Moreover, as $\eta_{0} S_{0}$ is smooth in $\Omega_{e} \backslash B\left(\boldsymbol{x}_{0}, \delta\right)$, applying the standard Green formula we get

$$
\left\langle\partial_{n}\left(\eta_{0} S_{0}\right), 1\right\rangle_{\Gamma_{0}}=\lim _{\delta \rightarrow 0}\left\langle\partial_{n}\left(\eta_{0} S_{0}\right), 1\right\rangle_{\Gamma_{0} \backslash[-\delta, \delta]} .
$$

Now for $r_{0}>\delta$, we compute $\partial_{n}\left(\eta_{0} S_{0}\right)$ separately on $\Gamma_{0}^{+}$and $\Gamma_{0}^{-}$. Denote by

$$
\boldsymbol{e}_{r}=\left(\begin{array}{c}
\cos \theta_{0} \\
\sin \theta_{0}
\end{array}\right), \boldsymbol{e}_{\theta}=\left(\begin{array}{c}
-\sin \theta_{0} \\
\cos \theta_{0}
\end{array}\right)
$$


the orthonormal basis associated with the local polar coordinates $\left(r_{0}, \theta_{0}\right)$. On $\Gamma_{0}^{+}$, we have $\boldsymbol{e}_{r} \cdot \boldsymbol{n}=0$ and $\boldsymbol{e}_{\theta} \cdot \boldsymbol{n}=+1$. Hence,

$$
\partial_{n}\left(\eta_{0} S_{0}\right)=\nabla\left(\eta_{0} S_{0}\right)_{\mid\left\{\theta_{0}=0\right\}} \cdot \boldsymbol{n}=\left(\eta_{0}^{\prime}\left(r_{0}\right) S_{0}\right)_{\left\{\theta_{0}=0\right\}} \boldsymbol{e}_{r} \cdot \boldsymbol{n}+\frac{1}{r_{0}} \eta_{0}\left(r_{0}\right)\left(\partial_{\theta} S_{0}\right)_{\left\{\theta_{0}=0\right\}} \boldsymbol{e}_{\theta} \cdot \boldsymbol{n}=\frac{1}{\omega_{e}} \frac{\eta_{0}\left(r_{0}\right)}{r_{0}} .
$$

On $\Gamma_{0}^{-}$, we have $\boldsymbol{e}_{r} \cdot \boldsymbol{n}=0$ and $\boldsymbol{e}_{\theta} \cdot \boldsymbol{n}=-1$. Therefore we get in a similar way,

$$
\partial_{n}\left(\eta_{0} S_{0}\right)=-\frac{1}{\omega_{e}} \frac{\eta_{0}\left(r_{0}\right)}{r_{0}}
$$

Using these expressions in (3.7) yields $\left\langle\partial_{n}\left(\eta_{0} S_{0}\right), 1\right\rangle_{\Gamma_{0}}=0$. Thus, $\left\langle\partial_{n} \varphi, 1\right\rangle_{\Gamma_{0}}=\left\langle\partial_{n} \varphi_{r}, 1\right\rangle_{\Gamma_{0}}=b$ which shows that $\varphi$ is a solution of (3.4). This solution is obviously unique.

It remains to estimate $\left\|\varphi_{r}\right\|_{1, \Omega_{e}}$ and $\left|c_{0}\right|$ in terms of the data $f, g$ and $b$. We have

$$
\|\tilde{f}\|_{0, \Omega_{e}} \lesssim\|f\|_{0, \Omega_{e}}+\left|\left[g\left(\boldsymbol{x}_{0}\right)\right]\right|
$$

since $\left\|\Delta\left(\eta_{0} S_{0}\right)\right\|_{0, \Omega_{e}}$ is bounded independently from $f, g$ and $b$. In order to estimate the term $\|\tilde{g}\|_{H^{1 / 2}\left(\Gamma_{0}\right) / \mathbb{C}}$, notice that

$$
\|\tilde{g}\|_{H^{1 / 2}\left(\Gamma_{0}\right) / \mathbb{C}} \leq\left\|\tilde{g}-\frac{1}{\operatorname{meas}\left(\Gamma_{0}\right)} \int_{\Gamma_{0}} \tilde{g} \mathrm{~d} \boldsymbol{x}\right\|_{1 / 2, \Gamma_{0}} \lesssim\left\|\partial_{s} \tilde{g}\right\|_{-1 / 2, \Gamma_{0}} .
$$

But $\partial_{s} \tilde{g}$ belongs to $L^{2}\left(\Gamma_{0}\right)$ since $\tilde{g}^{ \pm} \in H^{1}\left(\Gamma_{0}^{ \pm}\right)$and $\left[\tilde{g}\left(\boldsymbol{x}_{0}\right)\right]=0$. Thus,

$$
\left\|\partial_{s} \tilde{g}\right\|_{-1 / 2, \Gamma_{0}} \lesssim\left\|\partial_{s} \tilde{g}\right\|_{0, \Gamma_{0}} \lesssim\left\|\partial_{s} g^{+}\right\|_{0, \Gamma_{0}^{+}}+\left\|\partial_{s} g^{-}\right\|_{0, \Gamma_{0}^{-}}+\left|\left[g\left(\boldsymbol{x}_{0}\right)\right]\right| .
$$

Summing up, we get

$$
\left\|\varphi_{r}\right\|_{1, \Omega_{e}} \lesssim\|f\|_{0, \Omega_{e}}+\left\|\partial_{s} g\right\|_{0, \Gamma_{0}^{0}}+|b|+\left|\left[g\left(\boldsymbol{x}_{0}\right)\right]\right| .
$$

In order to estimate the jump $\left|c_{0}\right|=\left|\left[g\left(\boldsymbol{x}_{0}\right)\right]\right|$, let $g_{\Gamma_{0}}=\frac{1}{\operatorname{meas}\left(\Gamma_{0}\right)} \int_{\Gamma_{0}^{0}} g \mathrm{~d} \boldsymbol{x}$ be the mean value of $g$ on $\Gamma_{0}^{0}$. Then,

$$
\left|\left[g\left(\boldsymbol{x}_{0}\right)\right]\right|=\left|\left[g\left(\boldsymbol{x}_{0}\right)-g_{\Gamma_{0}}\right]\right| \lesssim\left\|g-g_{\Gamma_{0}}\right\|_{\infty, \Gamma_{0}^{0}} \lesssim\left\|g-g_{\Gamma_{0}}\right\|_{1, \Gamma_{0}^{0}}
$$

since the embedding from $H^{1}\left(\Gamma_{0}^{0}\right)$ into $\mathcal{C}^{0}\left(\overline{\Gamma_{0}^{ \pm}}\right)$is continuous. On the other hand, Poincaré-Wirtinger's inequality reads

$$
\left\|g-g_{\Gamma_{0}}\right\|_{0, \Gamma_{0}^{0}} \lesssim\left\|\partial_{s} g\right\|_{0, \Gamma_{0}^{0}}
$$

and thus

$$
\left|\left[g\left(\boldsymbol{x}_{0}\right)\right]\right| \lesssim\left\|\partial_{s} g\right\|_{0, \Gamma_{0}^{0}}
$$

This completes the proof.

Next, we define the weighted space of vector fields

$$
\mathbf{X}_{\alpha}(\Omega)=\left\{\begin{array}{l|l}
\boldsymbol{v} \in\left(\mathcal{D}^{\prime}(\Omega)\right)^{2} & \begin{array}{l}
\operatorname{curl} \boldsymbol{v} \in L^{2}(\Omega) ; \boldsymbol{v}_{c} \in \mathcal{H}_{0}\left(\operatorname{div} ; \Omega_{c}\right) ; \\
r_{0}^{\alpha} \boldsymbol{v}_{e} \in \mathbf{L}^{2}\left(\Omega_{e}\right) ; \operatorname{div} \boldsymbol{v}_{e} \in L^{2}\left(\Omega_{e}\right) ; \boldsymbol{v}_{e} \times \boldsymbol{n}=0 \quad \text { on } \quad \Gamma_{\infty} .
\end{array}
\end{array}\right\}
$$

$\mathbf{X}_{\alpha}(\Omega)$ is equipped with the following norm,

$$
\|\boldsymbol{v}\|_{\mathbf{X}_{\alpha}(\Omega)}=\left(\|\operatorname{curl} \boldsymbol{v}\|_{0, \Omega}^{2}+\left\|\boldsymbol{v}_{c}\right\|_{0, \Omega_{c}}^{2}+\left\|\operatorname{div} \boldsymbol{v}_{c}\right\|_{0, \Omega_{c}}^{2}+\left\|r_{0}^{\alpha} \boldsymbol{v}_{e}\right\|_{0, \Omega_{e}}^{2}+\left\|\operatorname{div} \boldsymbol{v}_{e}\right\|_{0, \Omega_{e}}^{2}\right)^{1 / 2}
$$


We now introduce the singular functions $S^{*}, S_{0}, S^{\mathrm{Neu}, \omega}$ and $S^{\mathrm{Dir}, \omega}$ which are defined in local polar coordinates by

$$
\begin{aligned}
S^{*}\left(r^{*}, \theta^{*}\right) & =\left(r^{*}\right)^{1 / 2} \sin \frac{\theta^{*}}{2}, \\
S_{0}\left(r_{0}, \theta_{0}\right) & =\frac{\theta_{0}}{\omega_{e}}, \\
S^{\mathrm{Neu}, \omega}\left(r_{0}, \theta_{0}\right) & =r_{0}^{\pi / \omega} \cos \left(\frac{\pi}{\omega}\left(\theta_{0}-\bar{\theta}\right)\right), \\
S^{\text {Dir }, \omega}\left(r_{0}, \theta_{0}\right) & =r_{0}^{\pi / \omega} \sin \left(\frac{\pi}{\omega} \theta_{0}\right) .
\end{aligned}
$$

Here, $S^{\mathrm{Neu}, \omega}$ and $S^{\mathrm{Dir}, \omega}$ are the standard singularities for the Laplace operator with Neumann and Dirichlet conditions, respectively, in a sector with opening angle $\omega>\pi$. Notice that we use the same local polar coordinates at $\boldsymbol{x}_{0}$ for $\Omega_{c}^{ \pm}$and $\Omega_{e}$. We introduce the variable $\bar{\theta}$ in the definition of $S^{\mathrm{Neu}, \omega}$ in order to be able to deal with both sides of the crack: $\bar{\theta}=0$ if $\omega=\omega_{c}^{+}$and $\bar{\theta}=\omega_{c}^{+}$if $\omega=\omega_{c}^{-}$.

We further define the function $S^{+}$(resp. $S^{-}$) as the variational solution in $H^{1}\left(\Omega_{e}\right)$ of the following problem with boundary data induced by the Neumann singularity $S^{\mathrm{Neu}, \omega_{c}^{+}}\left(\right.$resp. $\left.S^{\mathrm{Neu}, \omega_{c}^{-}}\right)$:

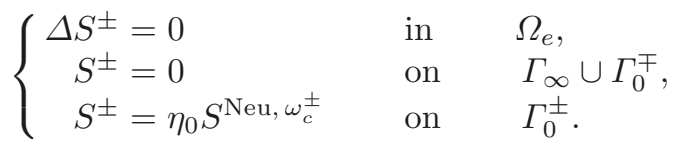

Notice that $S^{\mathrm{Neu}, \omega_{c}^{ \pm}}$vanishes for $r_{0}=0$. Therefore, the boundary data of (3.10) belong to $H^{1 / 2}\left(\Gamma_{0}\right)$ and $S^{ \pm}$ is well defined.

The electric field in the exterior domain $\Omega_{e}$ is allowed to present strong singularities at the crack mouth $\boldsymbol{x}_{0}$ since we only require that $r_{0}^{\alpha} \boldsymbol{v}_{e}$ belongs to $\boldsymbol{L}^{2}\left(\Omega_{e}\right)$ for a given positive weight parameter $\alpha$. The following theorem yields a decomposition of the elements in $\mathbf{X}_{\alpha}(\Omega)$ into a (piecewise) regular part and a singular part deriving from scalar potentials. These scalar potentials are localized near the crack tip $\boldsymbol{x}^{*}$ and the crack mouth $\boldsymbol{x}_{0}$.

Theorem 3.3. Assume that $0<\alpha<1 / 2$. Any field $\boldsymbol{v}$ in the space $\mathbf{X}_{\alpha}(\Omega)$ admits the decomposition

$$
\begin{aligned}
& \boldsymbol{v}_{c}=\boldsymbol{w}_{c}+c^{*} \nabla\left(\eta^{*} S^{*}\right)+c^{+} \nabla\left(\eta_{0} \mathbb{1}_{\mid \Omega_{c}^{+}} S^{\text {Neu, } \omega_{c}^{+}}\right)+c^{-} \nabla\left(\eta_{0} \mathbb{1}_{\mid \Omega_{c}^{-}} S^{\text {Neu, } \omega_{c}^{-}}\right) \text {in } \Omega_{c}, \\
& \boldsymbol{v}_{e}=\boldsymbol{w}_{e}+c_{0} \nabla\left(\eta_{0} S_{0}\right)+c^{+} \nabla S^{+}+c^{-} \nabla S^{-}+c_{e} \nabla\left(\eta_{0} S^{\text {Dir, } \omega_{e}}\right) \text { in } \Omega_{e}
\end{aligned}
$$

where $\boldsymbol{w}_{c}$ and $\boldsymbol{w}_{e}$ are regular fields in $\boldsymbol{H}^{1}\left(\Omega_{c}\right)$ and $\boldsymbol{H}^{1}\left(\Omega_{e}\right)$, respectively, and $c^{*}, c^{ \pm}, c_{0}$, and $c_{e}$ are complex numbers. We have $c^{+}=0$ if $\omega_{c}^{+} \leq \pi, c^{-}=0$ if $\omega_{c}^{-} \leq \pi$ and $c_{e}=0$ if $\omega_{e} \leq \pi$. The decomposition satisfies the estimates

$$
\left\|\boldsymbol{w}_{c}\right\|_{1, \Omega_{c}}+\left|c^{*}\right|+\left|c^{+}\right|+\left|c^{-}\right| \lesssim\left\|\operatorname{curl} \boldsymbol{v}_{c}\right\|_{0, \Omega_{c}}+\left\|\operatorname{div} \boldsymbol{v}_{c}\right\|_{0, \Omega_{c}}
$$

and

$$
\left\|\boldsymbol{w}_{e}\right\|_{1, \Omega_{e}}+\left|c_{0}\right|+\left|c_{e}\right| \lesssim\left\|\operatorname{curl} \boldsymbol{v}_{c}\right\|_{0, \Omega_{c}}+\left\|\operatorname{div} \boldsymbol{v}_{c}\right\|_{0, \Omega_{c}}+\left\|\operatorname{curl} \boldsymbol{v}_{e}\right\|_{0, \Omega_{e}}+\left\|\operatorname{div} \boldsymbol{v}_{e}\right\|_{0, \Omega_{e}}+\left|\left\langle\gamma_{n} \boldsymbol{v}_{e}, 1\right\rangle_{\Gamma_{0}}\right| .
$$

Proof. Let $\boldsymbol{v} \in \mathbf{X}_{\alpha}(\Omega)$. Since $\boldsymbol{v}_{c}$ belongs to $\mathcal{H}\left(\operatorname{curl} ; \Omega_{c}\right) \cap \mathcal{H}_{0}\left(\operatorname{div} ; \Omega_{c}\right)$, we deduce as in [31] that $\boldsymbol{v}_{c}$ admits the decomposition (3.11a) with $\boldsymbol{w}_{c} \in \boldsymbol{H}^{1}\left(\Omega_{c}\right), c^{*}, c^{ \pm} \in \mathbb{C}$ and $S^{*}$ (resp. $S^{\mathrm{Neu}, \omega_{c}^{ \pm}}$) defined by (3.9a) (resp. (3.9c)). Notice that the singularity at the crack mouth only occurs if $\Omega_{c}^{+}$(resp. $\Omega_{c}^{-}$) is not convex near $\boldsymbol{x}_{0}$. Since $\omega_{c}^{+}$ or $\omega_{c}^{-}$cannot both be larger than $\pi$, we assume without loss of generality from now on that $\omega_{c}^{-} \leq \pi$ so that $c^{-}=0$.

In order to prove estimate (3.12), consider the linear operator

$$
T:\left(\boldsymbol{H}^{1}\left(\Omega_{c}\right) \cap \mathcal{H}_{0}\left(\operatorname{div} ; \Omega_{c}\right)\right) \times \mathbb{C} \times \mathbb{C} \rightarrow \mathcal{H}\left(\operatorname{curl} ; \Omega_{c}\right) \cap \mathcal{H}_{0}\left(\operatorname{div} ; \Omega_{c}\right)
$$


defined by

$$
T\left(\boldsymbol{w}_{c}, c^{*}, c^{+}\right)=\boldsymbol{w}_{c}+c^{*} \nabla\left(\eta^{*} S^{*}\right)+c^{+} \nabla\left(\eta_{0} \mathbb{1}_{\mid \Omega_{c}^{+}} S^{\mathrm{Neu}, \omega_{c}^{+}}\right) .
$$

The operator is obviously continuous and one-to-one, and $T^{-1}$ is thus continuous owing to the closed graph theorem. Therefore,

$$
\left\|\boldsymbol{w}_{c}\right\|_{1, \Omega_{c}}+\left|c^{*}\right|+\left|c^{+}\right| \lesssim\left\|T\left(\boldsymbol{w}_{c}, c^{*}, c^{+}\right)\right\|_{\mathcal{H}\left(\operatorname{curl} ; \Omega_{c}\right) \cap \mathcal{H}_{0}\left(\operatorname{div} ; \Omega_{c}\right)}
$$

which proves (3.12) since the embedding of $\mathcal{H}\left(\operatorname{curl} ; \Omega_{c}\right) \cap \mathcal{H}_{0}\left(\operatorname{div} ; \Omega_{c}\right)$ into $\boldsymbol{L}^{2}\left(\Omega_{c}\right)$ is compact and the semi-norm $\left(\|\operatorname{curl} \cdot\|_{0, \Omega_{c}}^{2}+\|\operatorname{div} \cdot\|_{0, \Omega_{c}}^{2}\right)^{1 / 2}$ actually defines a norm on $\mathcal{H}\left(\operatorname{curl} ; \Omega_{c}\right) \cap \mathcal{H}_{0}\left(\operatorname{div} ; \Omega_{c}\right)$ equivalent to the canonical norm.

The decomposition of the exterior field $\boldsymbol{v}_{e}$ is less standard. From the definition of the space $\mathbf{X}_{\alpha}(\Omega)$, we get $\boldsymbol{v} \in \mathbf{L}_{\alpha}^{2}\left(\Omega_{e}\right)$, div $\boldsymbol{v} \in L^{2}\left(\Omega_{e}\right)$ and $\gamma_{t} \boldsymbol{v}_{e}=0$ on $\Gamma_{\infty}$. Moreover, we know that curl $\boldsymbol{v}$ belongs to $L^{2}$ on the whole domain $\Omega$ which implies the continuity of the tangential trace $\gamma_{t}$ across $\Gamma_{0}$ away from the crack mouth,

$$
\gamma_{t} \boldsymbol{v}_{e}=\left\{\begin{array}{lll}
\gamma_{t} \boldsymbol{w}_{c}+c^{+} \partial_{s}\left(\eta_{0} S^{\mathrm{Neu}, \omega_{c}^{+}}\right) & \text {on } & \Gamma_{0}^{+}, \\
\gamma_{t} \boldsymbol{w}_{c} & \text { on } & \Gamma_{0}^{-}
\end{array}\right.
$$

Notice further that $\boldsymbol{v}_{e}$ belongs to $\boldsymbol{L}^{p}\left(\Omega_{e}\right)$ for any $p$ such that $1 \leq p<\frac{2}{\alpha+1}$ according to Lemma 3.1. Owing to Corollary B.3, $\boldsymbol{v}_{e}$ thus admits the decomposition

$$
\boldsymbol{v}_{e}=\boldsymbol{v}_{e, r}+\nabla \varphi_{e}
$$

with $\boldsymbol{v}_{e, r} \in H^{1}\left(\Omega_{e}\right)$ such that $\gamma_{t} \boldsymbol{v}_{e, r}=0$ on $\Gamma_{\infty}$, and $\varphi_{e} \in W_{0, \Gamma_{\infty}}^{1, p}\left(\Omega_{e}\right)$. The a priori-estimate for $\boldsymbol{v}_{e, r}$ reads

$$
\left\|\boldsymbol{v}_{e, r}\right\|_{1, \Omega_{e}} \lesssim\left\|\operatorname{curl} \boldsymbol{v}_{e}\right\|_{0, \Omega_{e}}
$$

The scalar potential $\varphi_{e}$ admits a trace $\gamma_{0} \varphi_{e} \in W^{1-1 / p, p}\left(\Gamma_{0}\right)$. It follows from Sobolev's embedding theorem that $\gamma_{0} \varphi_{e}$ belongs to $L^{2}\left(\Gamma_{0}\right)$ since $W^{1-1 / p, p}\left(\Gamma_{0}\right) \hookrightarrow L^{2}\left(\Gamma_{0}\right)$ whenever $1-\frac{1}{p}>-\frac{1}{2}$ which yields $p>\frac{4}{3}$. We thus choose $p$ such that $\frac{4}{3}<p<\frac{2}{\alpha+1}$ which is possible since $\alpha<\frac{1}{2}$. From decomposition (3.15) and the continuity relation (3.14), we deduce that $\partial_{s} \varphi_{e}$ splits into a regular part and a singular part occurring from the lack of convexity of the interior domain $\Omega_{c}^{+}$at $\boldsymbol{x}_{0}$,

$$
\partial_{s} \varphi_{e}=\gamma_{t}\left(\nabla \varphi_{e}\right)=\gamma_{t}\left(\boldsymbol{v}_{e}-\boldsymbol{v}_{e, r}\right)= \begin{cases}\gamma_{t}\left(\boldsymbol{w}_{c}-\boldsymbol{v}_{e, r}\right)+c^{+} \partial_{s}\left(\eta_{0} S^{\mathrm{Neu}, \omega_{c}^{+}}\right) & \text {on } \Gamma_{0}^{+} \\ \gamma_{t}\left(\boldsymbol{w}_{c}-\boldsymbol{v}_{e, r}\right) & \text { on } \Gamma_{0}^{-}\end{cases}
$$

Now, notice that in the vicinity of $x_{0}, \partial_{s}\left(\eta_{0} S^{\text {Neu, } \omega_{c}^{-}}\right) \simeq s^{\lambda-1} \in L^{2}\left(\Gamma_{0}^{+}\right)$since $\lambda=\pi / \omega_{c}^{+}>1 / 2$. Hence $\varphi_{e}$ belongs to $H^{1}\left(\Gamma_{0}^{0}\right)$.

Summing up, the function $\varphi_{e}$ is the solution in $W_{0, \Gamma_{\infty}}^{1, p}\left(\Omega_{e}\right)$ of the following boundary value problem

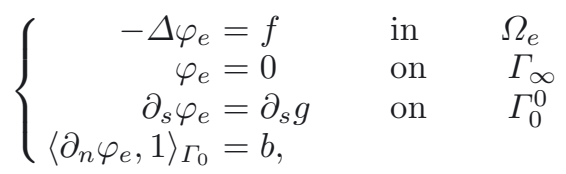

with $f=\operatorname{div}\left(\boldsymbol{v}_{e}-\boldsymbol{v}_{e, r}\right) \in L^{2}\left(\Omega_{e}\right), g \in H^{1}\left(\Gamma_{0}^{0}\right)$, and $b \in \mathbb{C}$. Notice that $\varphi_{e}$ is determined on $\Gamma_{0}^{0}$ up to an additive constant only. We deduce from Proposition 3.2 that $\varphi_{e}$ admits the decomposition

$$
\varphi_{e}=\varphi_{e, r}+c_{0} \eta_{0} S_{0}
$$

with $\varphi_{e, r} \in H^{1}\left(\Omega_{e}\right)$ and $c_{0} \in \mathbb{C}$. The decomposition satisfies the a priori-estimate

$$
\left\|\varphi_{e, r}\right\|_{1, \Omega_{e}}+\left|c_{0}\right| \lesssim\left\|\Delta \varphi_{e}\right\|_{0, \Omega_{e}}+\left\|\partial_{s} \varphi_{e}\right\|_{0, \Gamma_{0}^{0}}+\left|\left\langle\partial_{n} \varphi_{e}, 1\right\rangle_{\Gamma_{0}}\right| .
$$


According to the regularity results for $S_{0}$ stated in Proposition A.1, $\varphi_{e}$ belongs to the weighted space $H^{1, \alpha}\left(\Omega_{e}\right)$.

Now, notice that we get

$$
\begin{aligned}
\left\|\Delta \varphi_{e}\right\|_{0, \Omega_{e}} & \lesssim\left\|\operatorname{div} \boldsymbol{v}_{e, r}\right\|_{0, \Omega_{e}}+\left\|\operatorname{div} \boldsymbol{v}_{e}\right\|_{0, \Omega_{e}} \\
& \lesssim\left\|\operatorname{curl} \boldsymbol{v}_{e}\right\|_{0, \Omega_{e}}+\left\|\operatorname{div} \boldsymbol{v}_{e}\right\|_{0, \Omega_{e}}
\end{aligned}
$$

from estimate (3.16) for $\boldsymbol{v}_{e, r}$. Taking into account the estimate (3.12) for $\boldsymbol{w}_{c}$ and $\left|c^{+}\right|$, we get in a similar way

$$
\begin{aligned}
\left\|\partial_{s} \varphi_{e}\right\|_{0, \Gamma_{0}^{0}} & \lesssim\left\|\gamma_{t} \boldsymbol{w}_{c}\right\|_{0, \Gamma_{0}^{0}}+\left\|\gamma_{t} \boldsymbol{v}_{e, r}\right\|_{0, \Gamma_{0}}+\left|c^{+}\right| \\
& \lesssim\left\|\boldsymbol{w}_{c}\right\|_{1, \Omega_{c}}+\left\|\boldsymbol{v}_{e, r}\right\|_{1, \Omega_{e}}+\left|c^{+}\right| \\
& \lesssim\left\|\operatorname{curl} \boldsymbol{v}_{c}\right\|_{0, \Omega_{c}}+\left\|\operatorname{div} \boldsymbol{v}_{c}\right\|_{0, \Omega_{c}}+\left\|\operatorname{curl} \boldsymbol{v}_{e}\right\|_{0, \Omega_{e}} .
\end{aligned}
$$

In order to estimate the last term in (3.19), notice that

$$
\begin{aligned}
\left|\left\langle\partial_{n} \varphi_{e}, 1\right\rangle_{\Gamma_{0}}\right| & \lesssim\left|\left\langle\gamma_{n} \boldsymbol{v}_{e}, 1\right\rangle_{\Gamma_{0}}\right|+\left|\left\langle\gamma_{n} \boldsymbol{v}_{e, r}, 1\right\rangle_{\Gamma_{0}}\right| \\
& \lesssim\left|\left\langle\gamma_{n} \boldsymbol{v}_{e}, 1\right\rangle_{\Gamma_{0}}\right|+\left\|\boldsymbol{v}_{e, r}\right\|_{1, \Omega_{e}} \\
& \lesssim\left|\left\langle\gamma_{n} \boldsymbol{v}_{e}, 1\right\rangle_{\Gamma_{0}}\right|+\left\|\operatorname{curl} \boldsymbol{v}_{e}\right\|_{0, \Omega_{e}}
\end{aligned}
$$

where we used once again (3.16).

This leads to the estimate

$$
\left\|\varphi_{e, r}\right\|_{1, \Omega_{e}}+\left|c_{0}\right| \lesssim\left\|\operatorname{curl} \boldsymbol{v}_{c}\right\|_{0, \Omega_{c}}+\left\|\operatorname{div} \boldsymbol{v}_{c}\right\|_{0, \Omega_{c}}+\left\|\operatorname{curl} \boldsymbol{v}_{e}\right\|_{0, \Omega_{e}}+\left\|\operatorname{div} \boldsymbol{v}_{e}\right\|_{0, \Omega_{e}}+\left|\left\langle\gamma_{n} \boldsymbol{v}_{e}, 1\right\rangle_{\Gamma_{0}}\right|
$$

in terms of the vector field $\boldsymbol{v}$.

Notice that the function $\varphi_{e, r}$ obtained by the Helmholtz decomposition in Corollary B.3 has only $H^{1}$ regularity. In order to get a splitting into a regular part of class $H^{2}$ and an explicit singular part, we proceed in several steps: first, recall that the function $S^{+} \in H^{1}\left(\Omega_{e}\right)$ satisfies

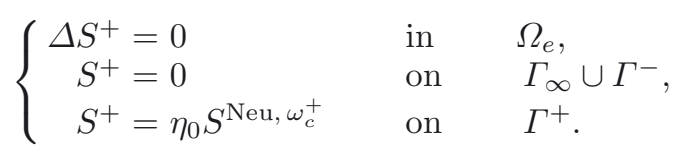

Next, set $\psi=\varphi_{e, r}-c^{+} S^{+} \in H^{1}\left(\Omega_{e}\right)$. From (3.17) and the definition of $\varphi_{e, r}$ we deduce that

$$
\partial_{s} \psi=\gamma_{t}\left(\boldsymbol{w}_{e}-\boldsymbol{v}_{e, r}\right)-c_{0} \partial_{s}\left(\eta_{0} S_{0}\right) \in H^{1 / 2}\left(\Gamma_{0}^{0}\right)
$$

which implies that $\gamma_{0} \psi \in H^{3 / 2}\left(\Gamma_{0}^{ \pm}\right)$on either side of $\boldsymbol{x}_{0}$. Notice that $\partial_{s}\left(\eta_{0} S_{0}\right)$ is regular and vanishes identically in a vicinity of $x_{0}$ since $S_{0}$ is piecewise constant on $\Gamma_{0}$. Then we claim that there is a continuous lifting $\chi \in H^{2}\left(\Omega_{e}\right) \cap H_{0, \Gamma_{\infty}}^{1}\left(\Omega_{e}\right)$ such that $\gamma_{0} \chi=\gamma_{0} \psi$ on $\Gamma_{0}$ and

$$
\|\chi\|_{2, \Omega_{e}} \lesssim\|\psi\|_{1 / 2, \Gamma_{0}}+\left\|\partial_{s} \psi\right\|_{1 / 2, \Gamma_{0}^{0}}
$$

Such a lifting exists whenever the data on either side of $\boldsymbol{x}_{0}$ are regular and satisfy appropriate compatibility conditions ( $c f$. [26]) between normal and tangential derivatives. In the present study, we only prescribe the trace of $\chi$ on $\Gamma_{0}$. The normal derivatives of $\chi$ on $\Gamma_{0}^{ \pm}$may thus be chosen in terms of the tangential derivatives in order to satisfy the compatibility conditions. The only difficulty occurs if $\omega_{e}=\pi$ since we have to ensure that

$$
\gamma_{t}\left(\boldsymbol{w}_{c}-\boldsymbol{v}_{e, r}\right)_{\mid \Gamma_{0}^{+}} \equiv \gamma_{t}\left(\boldsymbol{w}_{c}-\boldsymbol{v}_{e, r}\right)_{\mid \Gamma_{0}^{-}} \text {at } \boldsymbol{x}_{0} .
$$


To this end, we notice that the normal trace of $\boldsymbol{w}_{c}$ vanishes both on $\Gamma_{0}^{ \pm}$and $\Sigma$. Since $\boldsymbol{w}_{c}$ is regular near $\boldsymbol{x}_{0}$ on either side of $\Sigma$, its trace $\gamma_{0} \boldsymbol{w}_{c}$ is of class $H^{1 / 2}$. At $\boldsymbol{x}_{0},\left(\gamma_{0} \boldsymbol{w}_{c}\right)_{\mid \Gamma_{0}^{ \pm}}$is thus orthogonal to the two linear independent vectors, $\boldsymbol{n}_{\Sigma}$ and $\boldsymbol{n}_{\Gamma_{0}^{ \pm}}$. This implies

$$
\left(\gamma_{0} \boldsymbol{w}_{c}\right)_{\mid \Gamma_{0}^{ \pm}} \equiv \mathbf{0} \text { at } \boldsymbol{x}_{0}
$$

in the sense of $H^{1 / 2}$. The field $\boldsymbol{v}_{e, r}$ is regular in $\Omega_{e}$ and its trace belongs to $H^{1 / 2}\left(\Gamma_{0}\right)$. This proves (3.22) in the particular case $\omega_{e}=\pi$.

Finally, $\psi-\chi$ is the variational solution of a homogeneous Dirichlet problem. Therefore, $\psi-\chi$ admits the decomposition

$$
\psi-\chi=\psi_{r}+c_{e} \eta_{0} S^{\text {Dir, } \omega_{\mathrm{e}}}
$$

into a regular part $\psi_{r} \in H^{2}\left(\Omega_{e}\right) \cap H_{0}^{1}\left(\Omega_{e}\right)$ and a non-vanishing singular part whenever $\omega_{e}>\pi$.

Summing up, we obtain the following decomposition of $\varphi_{e}$

$$
\varphi_{e}=\psi_{r}+\chi+c_{e} \eta_{0} S^{\text {Dir }, \omega_{e}}+c^{+} S^{+}+c_{0} \eta_{0} S_{0} .
$$

Then, setting $\boldsymbol{w}_{e}=\boldsymbol{v}_{e, r}+\nabla\left(\psi_{r}+\chi\right) \in \boldsymbol{H}^{1}\left(\Omega_{e}\right)$ yields (3.11b).

The a priori-estimate (3.13) for $\boldsymbol{w}_{e}$ and the constants $c_{0}$ and $c_{e}$ follows from the continuity of decomposition (3.23), the continuity of the lifting (3.21), and estimates (3.20) and (3.16) for $\varphi_{e, r}$ and $\boldsymbol{v}_{e, r}$, respectively.

This completes the proof.

Let us make some remarks on the decomposition of Theorem 3.3. We see that the interior and exterior singular parts derive from scalar potentials which are given explicitly. At the crack tip, we recognize the standard singularity in $\sqrt{r}$. At the crack mouth, a singular behavior of Neumann type occurs inside the conductor whenever one of the opening angles on either side of the crack is larger than $\pi$. Outside the conductor, there is a new type of singularity coming from the discontinuity of the tangential trace at $\boldsymbol{x}_{0}$. In addition, a singularity of Dirichlet type occurs if the outer angle is larger than $\pi$, and, according to Lemma 3.4 hereafter, a "shadow" of the interior Neumann singularities can be observed.

We have the following decomposition of $S^{ \pm}$into a regular part belonging to $H^{2}$ and an explicit singular part:

Lemma 3.4. Assume that $\omega_{c}^{ \pm} \neq \omega_{e}$. There is a function $\varphi^{ \pm}=\varphi^{ \pm}\left(\theta_{0}\right)$ belonging to $\mathcal{C}^{\infty}\left(\left[-\omega_{e}, 0\right]\right)$, a function $S_{\mathrm{reg}}^{ \pm} \in H^{2}\left(\Omega_{e}\right)$ and $d^{ \pm} \in \mathbb{R}$ such that the solution $S^{ \pm}$of problem (3.10) may be written as

$$
S^{ \pm}=\eta_{0} r_{0}^{\pi / \omega_{c}^{ \pm}} \varphi^{ \pm}\left(\theta_{0}\right)+d^{ \pm} \eta_{0} S^{D i r, \omega_{e}}+S_{\text {reg }}^{ \pm}
$$

Proof. Let us prove the result for $S^{+}$. Notice that for $R_{0}$ small enough, we have

$$
S^{\mathrm{Neu}, \omega_{c}^{+}}=+r_{0}^{\pi / \omega_{c}^{+}} \text {on } \Gamma_{0}^{+} \cap\left\{r_{0}<R_{0}\right\} .
$$

At a first stage, we look for an explicit solution $\mathcal{S}^{+}$of

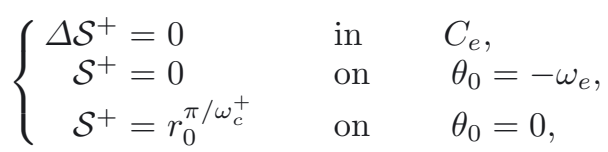

where $C_{e}$ is the infinite cone that coincides with $\Omega_{e}$ near the crack mouth. $\mathcal{S}^{+}$can be computed explicitly and is of the form

$$
\mathcal{S}^{+}\left(r_{0}, \theta_{0}\right)=r_{0}^{\pi / \omega_{c}^{+}} \varphi^{+}\left(\theta_{0}\right)
$$


The function $\varphi^{+}$solves the following one-dimensional problem,

$$
\left\{\begin{aligned}
\varphi^{\prime \prime}+\lambda^{2} \varphi & =0 \quad \text { in } \quad\left(-\omega_{e}, 0\right) \\
\varphi\left(-\omega_{e}\right) & =0 \\
\varphi(0) & =1
\end{aligned}\right.
$$

with $\lambda=\pi / \omega_{c}^{+}$. Such a solution exists since $\omega_{c}^{+} \neq \omega_{e}$ and thus, $\lambda^{2}$ is not an eigenvalue of the operator $-\frac{d^{2}}{\mathrm{~d} \theta_{0}^{2}}$ with Dirichlet condition in the interval $\left(-\omega_{e}, 0\right)$. More precisely, $\varphi^{+}$belongs to $\mathcal{C}^{\infty}\left(\left[-\omega_{e}, 0\right]\right)$ and reads

$$
\varphi^{+}\left(\theta_{0}\right)=A \sin \lambda \theta_{0}+B \cos \lambda \theta_{0}
$$

with real coefficients $A$ and $B$ that can be uniquely determined from the boundary conditions.

At a second stage, we split $S^{+}$into

$$
S^{+}=\eta_{0} \mathcal{S}^{+}+R
$$

and notice that $R \in H_{0}^{1}\left(\Omega_{e}\right)$ is the solution of

$$
\Delta R=-\Delta\left(\eta_{0} \mathcal{S}^{+}\right) \text {in } \Omega_{e} .
$$

As this right-hand side belongs to $L^{2}\left(\Omega_{e}\right)$, it follows from standard regularity results (see for instance [25]) that $R$ admits the splitting

$$
R=d^{+} \eta_{0} S^{\mathrm{Dir}, \omega_{e}}+S_{\text {reg }}^{+},
$$

with $S_{\text {reg }}^{+} \in H^{2}\left(\Omega_{e}\right)$ and a real number $d^{+}$. This proves (3.25).

Notice that the condition $\omega_{c}^{ \pm} \neq \omega_{e}$ is not restrictive in the present setting. Indeed, $\omega_{c}^{+}=\omega_{e}$ implies that $\omega_{c}^{+}<\pi$ since $\omega_{c}^{-}>0$. Therefore, no interior singularity occurs at $x_{0}$ in $\Omega_{c}^{+}$and the coefficient $c^{+}$in decomposition (3.11) vanishes. This means that there is no need for a decomposition of $S^{+}$. The case $\omega_{c}^{-}=\omega_{e}$ can be handled in the same way.

\section{VARIATIONAL FORMUlations of THE EDDY-CURRENT PROBLEM}

\subsection{The electric field formulation}

Consider the following subspace of $\mathbf{X}_{\alpha}(\Omega)$,

$$
\mathbf{Y}_{\alpha}(\Omega)=\left\{\boldsymbol{v} \in \mathbf{X}_{\alpha}(\Omega) \mid\left\langle\gamma_{n} \boldsymbol{v}_{e}, 1\right\rangle_{\Gamma_{0}}=0\right\}
$$

The regularized variational formulation of problem (2.8) and (2.9) reads

$$
\left\{\begin{array}{l}
\text { Find } \boldsymbol{E} \in \mathbf{Y}_{\alpha}(\Omega) \text { such that } \\
a_{R}(\boldsymbol{E}, \boldsymbol{v})=-i \omega\left(\boldsymbol{J}_{s}, \boldsymbol{v}\right) \forall \boldsymbol{v} \in \mathbf{Y}_{\alpha}(\Omega),
\end{array}\right.
$$

where

$$
a_{R}(\boldsymbol{u}, \boldsymbol{v})=a_{0}(\boldsymbol{u}, \boldsymbol{v})+\int_{\Omega_{c}} \operatorname{div} \boldsymbol{u}_{c} \operatorname{div} \overline{\boldsymbol{v}}_{c} \mathrm{~d} \boldsymbol{x}+\int_{\Omega_{e}} \operatorname{div} \boldsymbol{u}_{e} \operatorname{div} \overline{\boldsymbol{v}}_{e} \mathrm{~d} \boldsymbol{x}
$$

with

$$
a_{0}(\boldsymbol{u}, \boldsymbol{v})=\int_{\Omega} \mu^{-1} \operatorname{curl} \boldsymbol{u} \operatorname{curl} \overline{\boldsymbol{v}} \mathrm{d} \boldsymbol{x}+i \omega \int_{\Omega_{c}} \sigma_{c} \boldsymbol{u} \cdot \overline{\boldsymbol{v}} \mathrm{d} \boldsymbol{x} .
$$

Theorem 4.1. Let $\boldsymbol{J}_{s} \in \boldsymbol{L}^{2}(\Omega)$ such that $\operatorname{div} \boldsymbol{J}_{s}=0$ in $\Omega$ and $\operatorname{supp}\left(\boldsymbol{J}_{s}\right) \subset \Omega_{e}$. Let $0<\alpha<1 / 2$. Then problem (4.2) has a unique solution $\boldsymbol{E}$ in $\mathbf{Y}_{\alpha}(\Omega)$. Moreover, $\boldsymbol{E}$ is divergence-free in $\Omega_{c}$ and $\Omega_{e}$.

The proof of Theorem 4.1 follows the ideas of [20] where an uncracked domain has been considered. 
Proof. We have

$$
\mathfrak{R} e\left(\mathrm{e}^{-\mathrm{i} \frac{\pi}{4}} a_{R}(\boldsymbol{u}, \boldsymbol{u})\right) \gtrsim\|\operatorname{curl} \boldsymbol{u}\|_{0, \Omega}^{2}+\left\|\operatorname{div} \boldsymbol{u}_{c}\right\|_{0, \Omega_{c}}^{2}+\left\|\operatorname{div} \boldsymbol{u}_{e}\right\|_{0, \Omega_{e}}^{2}+\left\|\boldsymbol{u}_{c}\right\|_{0, \Omega_{c}}^{2} .
$$

It thus remains to prove that the right hand side is an upper bound for the weighted norm $\left\|r_{0}^{\alpha} \boldsymbol{u}_{e}\right\|_{0, \Omega_{e}}$. According to Theorem 3.3, $\boldsymbol{u}_{e}$ splits into a regular and a singular part,

$$
\boldsymbol{u}_{e}=\boldsymbol{w}_{e}+c_{0} \nabla\left(\eta_{0} S_{0}\right)+c^{+} \nabla S^{+}+c^{-} \nabla S^{-}+c_{e} \nabla\left(\eta_{0} S^{\mathrm{Dir}, \omega_{e}}\right) .
$$

Therefore,

$$
\left\|r_{0}^{\alpha} \boldsymbol{u}_{e}\right\|_{0, \Omega_{e}}^{2} \lesssim\left\|\boldsymbol{w}_{e}\right\|_{0, \Omega_{e}}^{2}+\left|c_{0}\right|^{2}+\left|c^{+}\right|^{2}+\left|c^{-}\right|^{2}+\left|c_{e}\right|^{2}
$$

since $\boldsymbol{w}_{e} \in \boldsymbol{H}^{1}\left(\Omega_{e}\right), r_{0}^{\alpha} \leq M$ on $\Omega_{e}$ and $\left\|r_{0}^{\alpha} \nabla\left(\eta_{0} S_{0}\right)\right\|_{0, \Omega_{e}},\left\|r_{0}^{\alpha} \nabla S^{ \pm}\right\|_{0, \Omega_{e}}$, and $\left\|r_{0}^{\alpha} \nabla\left(\eta_{0} S^{\text {Dir, } \omega_{e}}\right)\right\|_{0, \Omega_{e}}$ are bounded independently from $\boldsymbol{u}$. It follows from estimates (3.12) and (3.13) that $a_{R}(\cdot, \cdot)$ is coercive on $\mathbf{Y}_{\alpha}(\Omega)$ since $\left\langle\gamma_{n} \boldsymbol{u}_{e}, 1\right\rangle_{\Gamma_{0}}=0$.

In order to prove that the solution $\boldsymbol{E}$ is divergence-free in $\Omega_{e}$, let $g_{e} \in L^{2}\left(\Omega_{e}\right)$ and consider the problem

$$
\left\{\begin{array}{l}
\text { Find } \varphi_{e} \in \Theta_{0} \text { such that } \\
\int_{\Omega_{e}} \nabla \varphi_{e} \cdot \nabla \bar{\psi}_{e} \mathrm{~d} \boldsymbol{x}=\int_{\Omega_{e}} g_{e} \bar{\psi}_{e} \mathrm{~d} \boldsymbol{x} \forall \psi_{e} \in \Theta_{0},
\end{array}\right.
$$

where

$$
\Theta_{0}=\left\{\psi \in H^{1}\left(\Omega_{e}\right) \mid \varphi=\text { const. on } \Gamma_{0}, \varphi=0 \text { on } \Gamma_{\infty}\right\} .
$$

$\Theta_{0}$ is obviously a closed subspace of $H^{1}\left(\Omega_{e}\right)$ on which Poincaré's inequality holds true. Thus, problem (4.3) has a unique solution due to Lax-Milgram's Lemma. It can easily be shown that the solution of (4.3) satisfies the following strong formulation.

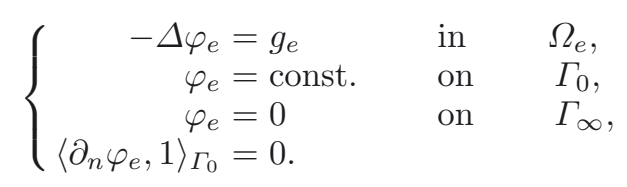

Now, define the vector field

$$
\boldsymbol{v}= \begin{cases}0 & \text { in } \Omega_{c} \\ \nabla \varphi_{e} & \text { in } \Omega_{e}\end{cases}
$$

Obviously, $\boldsymbol{v}$ belongs to $\mathbf{Y}_{\alpha}(\Omega)$ and is thus an admissible test field in (4.2). The variational equation reads

$$
\int_{\Omega_{e}} \operatorname{div} \boldsymbol{E}_{e} \Delta \bar{\varphi}_{e} \mathrm{~d} \boldsymbol{x}=-i \omega \int_{\Omega_{e}} \boldsymbol{J}_{s} \cdot \nabla \bar{\varphi}_{e} \mathrm{~d} \boldsymbol{x} .
$$

But the term on the right hand side vanishes since $\boldsymbol{J}_{s}$ is divergence-free and $\operatorname{supp}\left(\boldsymbol{J}_{s}\right) \subset \Omega_{e}$. Therefore,

$$
\int_{\Omega_{e}} \operatorname{div} \boldsymbol{E}_{e} g_{e} \mathrm{~d} \boldsymbol{x}=0
$$

for any $g_{e} \in L^{2}\left(\Omega_{e}\right)$ which proves that $\operatorname{div} \boldsymbol{E}_{e}=0$ in $\Omega_{e}$.

We show in a similar way that $\operatorname{div} \boldsymbol{E}_{c}=0$ in $\Omega_{c}$ since the operator $\Delta-i \omega \sigma_{c} \mathbb{I}$ is onto as an operator from $\left\{\psi \in H^{1}\left(\Omega_{c}\right) \mid \Delta \psi \in L^{2}\left(\Omega_{c}\right) ; \partial_{n} \psi=0\right.$ on $\left.\partial \Omega_{c}\right\}$ on $L^{2}\left(\Omega_{c}\right)$. Indeed, let $g_{c} \in L^{2}\left(\Omega_{c}\right)$ and consider the solution of the problem

$$
\left\{\begin{array}{l}
\text { Find } \varphi_{c} \in H^{1}\left(\Omega_{c}\right) \text { such that } \\
\int_{\Omega_{c}} \nabla \varphi_{c} \cdot \nabla \overline{\psi_{c}} \mathrm{~d} \boldsymbol{x}+i \omega \sigma_{c} \int_{\Omega_{c}} \varphi_{c} \overline{\psi_{c}} \mathrm{~d} \boldsymbol{x}=\int_{\Omega_{c}} g_{c} \overline{\psi_{c}} \mathrm{~d} \boldsymbol{x} \forall \psi_{c} \in H^{1}\left(\Omega_{c}\right)
\end{array}\right.
$$


The bilinear form of the above variational formulation is coercive on $H^{1}\left(\Omega_{c}\right)$ since

$$
\mathfrak{R} e\left(\mathrm{e}^{-\mathrm{i} \frac{\pi}{4}}\left(\left\|\nabla \psi_{c}\right\|_{0, \Omega_{c}}^{2}+i \omega \sigma_{c}\left\|\psi_{c}\right\|_{0, \Omega_{c}}^{2}\right)\right) \gtrsim\left\|\psi_{c}\right\|_{1, \Omega_{c}}^{2} \forall \psi_{c} \in H^{1}\left(\Omega_{c}\right) .
$$

Notice that $\varphi_{c}$ is of class $H^{3 / 2+\varepsilon}$ in $\Omega_{c}$ outside a neighborhood of the crack tip $\boldsymbol{x}^{*}$. In particular, this means that the trace $\left(\gamma_{0} \varphi_{c}\right)_{\mid \Gamma_{0}}$ belongs to $H^{1}\left(\Gamma_{0}^{0}\right)$, but $\left(\gamma_{0} \varphi_{c}\right)_{\mid \Gamma_{0}}$ may be discontinuous at the crack mouth $\boldsymbol{x}_{0}$. According to Proposition 3.2, there is an extension $\widetilde{\varphi_{c}} \in H^{1, \alpha}\left(\Omega_{e}\right)$ such that

$$
\gamma_{0} \widetilde{\varphi_{c}}=\gamma_{0} \varphi_{c}+d \text { on } \Gamma_{0}^{0}
$$

with a constant $d \in \mathbb{C}$. Then, the field

$$
\boldsymbol{v}=\left\{\begin{array}{lll}
\nabla \varphi_{c} & \text { in } & \Omega_{c} \\
\nabla \widetilde{\varphi_{c}} & \text { in } & \Omega_{e}
\end{array}\right.
$$

is admissible in (4.2). We get

$$
\int_{\Omega_{c}} \operatorname{div} \boldsymbol{E}_{c} \Delta \overline{\varphi_{c}} \mathrm{~d} \boldsymbol{x}+i \omega \sigma_{c} \int_{\Omega_{c}} \boldsymbol{E}_{c} \cdot \nabla \overline{\varphi_{c}} \mathrm{~d} \boldsymbol{x}=-i \omega \int_{\Omega_{e}} \boldsymbol{J}_{s} \cdot \nabla \overline{\overline{\varphi_{c}}} \mathrm{~d} \boldsymbol{x}
$$

which reads

$$
\int_{\Omega_{c}} \operatorname{div} \boldsymbol{E}_{c}\left(\Delta-i \omega \sigma_{c} \mathbb{I}\right) \overline{\varphi_{c}} \mathrm{~d} \boldsymbol{x}=0
$$

since $\boldsymbol{E}_{c} \cdot \boldsymbol{n}=0$ on $\partial \Omega_{c}$ and $\boldsymbol{J}_{s}$ is divergence-free and vanishes near the boundary of $\Omega_{e}$. Finally we have

$$
\int_{\Omega_{c}} \operatorname{div} \boldsymbol{E}_{c} g_{c} \mathrm{~d} \boldsymbol{x}=0
$$

for any $g_{c} \in L^{2}\left(\Omega_{c}\right)$ which completes the proof.

\subsection{The $A-\psi$-formulation}

The variational formulation (4.2) is not well suited for the effective computation of the electric field in $\Omega_{c}$ and $\Omega_{e}$. A classical way to compute eddy currents in the conductor $\Omega_{c}$ and the electric field in the exterior domain $\Omega_{e}$ is given by the $\boldsymbol{A}-\psi$-formulation which is formulated in terms of a magnetic vector potential $\boldsymbol{A}$ and a scalar potential $\psi$. To this end, notice that the normal magnetic flux $\boldsymbol{B} \cdot \boldsymbol{n}$ is continuous across the crack [11] and $\boldsymbol{B}$ is thus divergence-free in the uncracked domain $Q$. We introduce a vector potential $\boldsymbol{A} \in \mathcal{H}_{0}(\operatorname{curl} ; Q)$ such that

$$
\boldsymbol{B}=\mu \boldsymbol{H}=\operatorname{curl} \boldsymbol{A} .
$$

Notice that $\boldsymbol{A}$ can be chosen in $\boldsymbol{H}^{1}(Q)$ since $Q$ is regular.

From (2.1a), we get

$$
\operatorname{curl}(i \omega \boldsymbol{A}+\boldsymbol{E})=0 \text { in } \Omega .
$$

If $\boldsymbol{E}$ denotes the solution of the electric field formulation in $\mathbf{Y}_{\alpha}(\Omega)$, we know that $\boldsymbol{E}_{c} \in \boldsymbol{L}^{2}\left(\Omega_{c}\right)$ and $\boldsymbol{E}_{e} \in \boldsymbol{L}^{p}\left(\Omega_{e}\right)$ for any $1 \leq p<\frac{2}{\alpha+1}$. From Theorem B.2 and standard results in $L^{2}$, we infer the existence of a scalar potential $\psi$ such that

$$
\boldsymbol{E}=-i \omega(\boldsymbol{A}+\nabla \psi) \text { in } \Omega
$$

where $\psi_{c} \in H^{1}\left(\Omega_{c}\right)$ and $\psi_{e} \in W_{0, \Gamma_{\infty}}^{1, p}\left(\Omega_{e}\right)$. Moreover, Theorem 3.3 and the regularity of $\boldsymbol{A}$ imply that $\psi$ belongs to the weighted space $H^{1, \alpha}\left(\Omega_{e}\right)$. The continuity of the tangential traces across the interface $\Gamma_{0}$ of both $\boldsymbol{A}$ and $\boldsymbol{E}$ 
outside the crack mouth yields $\psi_{c}=\psi_{e}+d$ on $\Gamma_{0}^{0}$ for some constant $d \in \mathbb{C}$. The scalar potential $\psi$ thus belongs to the space

$$
\left\{\varphi \in L^{2}(\Omega) \mid \varphi_{c} \in H^{1}\left(\Omega_{c}\right) ; \varphi_{e} \in H^{1, \alpha}\left(\Omega_{e}\right) ; \varphi_{c}-\varphi_{e}=\text { const. on } \Gamma_{0}^{0} ; \varphi=0 \text { on } \Gamma_{\infty}\right\} .
$$

Taking into account that $\boldsymbol{J}=\sigma \boldsymbol{E}+\boldsymbol{J}_{s}$, we get from the latter identity and (2.1b),

$$
\operatorname{curl} \mu^{-1} \operatorname{curl} \boldsymbol{A}+i \omega \sigma(\boldsymbol{A}+\nabla \psi)=\boldsymbol{J}_{s} \text { in } \Omega
$$

completed by the divergence constraint

$$
i \omega \operatorname{div}(\sigma(\boldsymbol{A}+\nabla \psi))=0 \text { in } \Omega
$$

Notice that (4.6) and (4.7) define the scalar potential $\psi$ only in the conductor $\Omega_{c}$, whereas the vector potential $\boldsymbol{A}$ is given on the whole computational domain. We complete (4.6) and (4.7) by the boundary condition $\boldsymbol{A} \times \boldsymbol{n}=0$ on the outer boundary $\Gamma_{\infty}$. We further notice that $\boldsymbol{J} \cdot \boldsymbol{n}=0$ on $\Gamma_{0}$, as well as on the crack $\Sigma$. This follows from (4.7) since the conductivity is zero outside the conductor and (4.7) ensures the continuity of the normal component of $\boldsymbol{J}=-i \omega \sigma(\boldsymbol{A}+\nabla \psi)+\boldsymbol{J}_{s}$.

In this section, we aim to prove existence and uniqueness results for the $\boldsymbol{A}-\psi$-formulation. To this end, we need appropriate gauge conditions on the potentials $\boldsymbol{A}$ and $\psi$. Here, we choose the classical Coulomb gauge for the vector potential,

$$
\operatorname{div} \boldsymbol{A}=0 \text { in } Q .
$$

More precisely, the functional space for the vector potential is given by

$$
\boldsymbol{X}^{0}(Q)=\left\{\boldsymbol{v} \in \mathcal{H}_{0}(\operatorname{curl} ; Q) \mid(\boldsymbol{A}, \nabla \xi)=0 \forall \xi \in H_{0}^{1}(Q)\right\} .
$$

In order to fix the additive constant of the scalar potential $\psi$, we assume that $\psi$ is of zero mean value,

$$
\int_{\Omega_{c}} \psi \mathrm{d} \boldsymbol{x}=0
$$

Hence, $\psi$ is sought in the space

$$
U^{0}\left(\Omega_{c}\right)=H^{1}\left(\Omega_{c}\right) \cap L_{0}^{2}\left(\Omega_{c}\right)
$$

where $L_{0}^{2}\left(\Omega_{c}\right)$ is the subspace of $L^{2}\left(\Omega_{c}\right)$ of functions such that (4.10) holds true.

The variational formulation of the eddy current problem in the $\boldsymbol{A}-\psi$-formulation then reads

$$
\left\{\begin{array}{c}
\text { Find }(\boldsymbol{A}, \psi) \in \boldsymbol{X}^{0}(Q) \times U^{0}\left(\Omega_{c}\right) \text { such that } \\
\int_{\Omega} \mu^{-1} \operatorname{curl} \boldsymbol{A} \operatorname{curl} \overline{\boldsymbol{A}^{\prime}} \mathrm{d} \boldsymbol{x}+i \omega \int_{\Omega_{c}} \sigma_{c} \boldsymbol{A} \cdot \overline{\boldsymbol{A}^{\prime}} \mathrm{d} \boldsymbol{x}+i \omega \int_{\Omega_{c}} \sigma_{c} \nabla \psi \cdot \overline{\boldsymbol{A}^{\prime}} \mathrm{d} \boldsymbol{x}=\int_{\Omega_{e}} \boldsymbol{J}_{s} \cdot \overline{\boldsymbol{A}^{\prime}} \mathrm{d} \boldsymbol{x}, \forall \boldsymbol{A}^{\prime} \in \boldsymbol{X}^{0}(Q) \\
i \omega \int_{\Omega_{c}} \sigma_{c} \boldsymbol{A} \cdot \nabla \overline{\psi^{\prime}} \mathrm{d} \boldsymbol{x}+i \omega \int_{\Omega_{c}} \sigma_{c} \nabla \psi \cdot \nabla \overline{\psi^{\prime}} \mathrm{d} \boldsymbol{x}=0 \forall \psi^{\prime} \in U^{0}\left(\Omega_{c}\right) .
\end{array}\right.
$$

In order to prove that problem (4.12) is well posed, we follow the ideas in [21] where the $\boldsymbol{A}$ - $\psi$-formulation has been studied in the case of a non cracked domain. We introduce the vector space $V=\boldsymbol{X}^{0}(Q) \times U^{0}\left(\Omega_{c}\right)$ equipped with the norm

$$
\|(\boldsymbol{A}, \psi)\|_{V}=\left(\|\boldsymbol{A}\|_{\mathcal{H}(\operatorname{curl} ; Q)}^{2}+|\psi|_{1, \Omega_{c}}^{2}\right)^{1 / 2} .
$$

Here, $\|\cdot\|_{\mathcal{H}(\operatorname{curl} ; Q)}$ denotes the usual norm on $\mathcal{H}(\operatorname{curl} ; Q)$ whereas $|\cdot|_{1, \Omega_{c}}$ is the semi-norm on $H^{1}\left(\Omega_{c}\right)$ which actually defines a norm on $U^{0}\left(\Omega_{c}\right)$.

Then, (4.12) is equivalent to the variational problem

$$
\left\{\begin{array}{l}
\text { Find }(\boldsymbol{A}, \psi) \in V \text { such that } \\
a\left((\boldsymbol{A}, \psi),\left(\boldsymbol{A}^{\prime}, \psi^{\prime}\right)\right)=\ell\left(\left(\boldsymbol{A}^{\prime}, \psi^{\prime}\right)\right) \forall\left(\boldsymbol{A}^{\prime}, \psi^{\prime}\right) \in V
\end{array}\right.
$$


where the sesqui-linear form $a(\cdot, \cdot)$ is defined on $V \times V$ by

$$
a\left((\boldsymbol{A}, \psi),\left(\boldsymbol{A}^{\prime}, \psi^{\prime}\right)\right)=\int_{\Omega} \mu^{-1} \operatorname{curl} \boldsymbol{A} \operatorname{curl} \overline{\boldsymbol{A}^{\prime}} \mathrm{d} \boldsymbol{x}+i \omega \int_{\Omega_{c}} \sigma_{c}(\boldsymbol{A}+\nabla \psi) \cdot\left(\overline{\boldsymbol{A}^{\prime}}+\nabla \overline{\psi^{\prime}}\right) \mathrm{d} \boldsymbol{x}
$$

and the linear form $\ell(\cdot)$ is given on $V$ by

$$
\ell\left(\left(\boldsymbol{A}^{\prime}, \psi^{\prime}\right)\right)=\int_{\Omega_{e}} \boldsymbol{J}_{s} \cdot \overline{\boldsymbol{A}^{\prime}} \mathrm{d} \boldsymbol{x} .
$$

The coercivity of the sesqui-linear form $a(\cdot, \cdot)$ follows from an inequality of Friedrichs-Poincaré type for the vector potential $\boldsymbol{A}$ :

$$
\|\boldsymbol{A}\|_{0, Q} \lesssim\|\operatorname{curl} \boldsymbol{A}\|_{0, Q} \forall \boldsymbol{A} \in \boldsymbol{X}^{0}(Q) .
$$

Notice that $\boldsymbol{A}$ is sought in the same space as in [21] in spite of the presence of the crack.

The previous results allow to prove that problem (4.13) is well posed.

Theorem 4.2. Let $\boldsymbol{J}_{s} \in L^{2}(\Omega)$ such that $\operatorname{div} \boldsymbol{J}_{s}=0$ in $\Omega$. Assume further that $\operatorname{supp}\left(\boldsymbol{J}_{s}\right) \subset \Omega_{e}$. Then, the variational problem (4.13) has a unique solution $(\boldsymbol{A}, \psi) \in V$.

Proof. The sesqui-linear form $a(\cdot, \cdot)$ and the linear form $\ell(\cdot)$ are obviously continuous on the Hilbert space $V$. Notice further that

$$
\mathfrak{R} e\left(\mathrm{e}^{-i \frac{\pi}{4}} a((\boldsymbol{A}, \psi),(\boldsymbol{A}, \psi))\right)=\cos \frac{\pi}{4} \int_{\Omega} \mu^{-1}|\operatorname{curl} \boldsymbol{A}|^{2} \mathrm{~d} \boldsymbol{x}+\omega \sin \frac{\pi}{4} \int_{\Omega_{c}} \sigma_{c}|\boldsymbol{A}+\nabla \psi|^{2} \mathrm{~d} \boldsymbol{x} .
$$

We prove that the right hand side of the above identity is bounded from below by $\|(\boldsymbol{A}, \psi)\|_{V}^{2}$ up to a multiplicative constant by the same contradiction argument as in [21] with minor changes in the constants. This yields the coercivity of the sesqui-linear form $a(\cdot, \cdot)$ since

$$
|a((\boldsymbol{A}, \psi),(\boldsymbol{A}, \psi))| \geq \mathfrak{R} e\left(\mathrm{e}^{-i \frac{\pi}{4}} a((\boldsymbol{A}, \psi),(\boldsymbol{A}, \psi))\right) \geq \alpha\|(\boldsymbol{A}, \psi)\|_{V}^{2}
$$

for some positive constant $\alpha$ independent from $(\boldsymbol{A}, \psi)$. Consequently, problem (4.13) has a unique solution by Lax-Milgram's Lemma.

Actually, the solution of problem (4.13) satisfies the variational equation for test fields that do not fulfill the gauge conditions. The proof is in any way the same as in [21] since the presence of the crack only affects the definition of the scalar potential.

Proposition 4.3. Let $\boldsymbol{J}_{s} \in \boldsymbol{L}^{2}(\Omega)$ such that $\operatorname{div} \boldsymbol{J}_{s}=0$ in $\Omega$ and $\operatorname{supp}\left(\boldsymbol{J}_{s}\right) \subset \Omega_{e}$. Let $(\boldsymbol{A}, \psi) \in V$ be the unique solution of (4.13). Then

$$
a\left((\boldsymbol{A}, \psi),\left(\boldsymbol{A}^{\prime}, \psi^{\prime}\right)\right)=\ell\left(\left(\boldsymbol{A}^{\prime}, \psi^{\prime}\right)\right) \forall\left(\boldsymbol{A}^{\prime}, \psi^{\prime}\right) \in \mathcal{H}_{0}(\operatorname{curl} ; Q) \times H^{1}\left(\Omega_{c}\right) .
$$

\subsection{Equivalence between the two formulations}

In this section, we aim to prove equivalence between the variational formulation (4.2) for the electric field and the $\boldsymbol{A}-\psi$-formulation (4.13). The result is stated in the next theorem.

Theorem 4.4. Let $\boldsymbol{J}_{s} \in \boldsymbol{L}^{2}(\Omega)$ be such that $\operatorname{div} \boldsymbol{J}_{s}=0$ in $\Omega$ and $\operatorname{supp}\left(\boldsymbol{J}_{s}\right) \subset \Omega_{e}$. If $\boldsymbol{E} \in \mathbf{Y}_{\alpha}(\Omega)$ is the solution of (4.2), then there exists $\boldsymbol{A} \in \boldsymbol{X}^{0}(Q)$ and $\psi \in L^{2}(\Omega)$, such that $\left(\boldsymbol{A}, \psi_{c}\right)$ is solution of problem (4.13) and

$$
\boldsymbol{E}=-i \omega(\boldsymbol{A}+\nabla \psi) \text { in } \Omega .
$$

Conversely, if $(\boldsymbol{A}, \psi) \in V$ is solution of (4.13), then there is an extension of $\psi$ to $\Omega_{e}$, still denoted by $\psi$, such that $\boldsymbol{E}=-i \omega(\boldsymbol{A}+\nabla \psi)$ is solution of (4.2). 
Proof. Let $\left(\boldsymbol{A}, \psi_{c}\right) \in V$ be the unique solution of the gauged formulation (4.13). Since $\boldsymbol{A}$ is divergence-free in $Q$, it follows from the second equation of (4.12) that $\psi_{c} \in H^{1}\left(\Omega_{c}\right)$ is the solution of the Neumann problem

$$
\left\{\begin{array}{lll}
\Delta \psi_{c}=0 & \text { in } & \Omega_{c} \\
\partial_{n} \psi_{c}=\boldsymbol{A} \cdot n & \text { on } & \partial \Omega_{c} .
\end{array}\right.
$$

As $Q$ has a $C^{1,1}$ boundary, it is well known that $\boldsymbol{X}^{0}(Q) \hookrightarrow \boldsymbol{H}^{1}(Q)$ (see for instance [24]) so that $\boldsymbol{A} \in \boldsymbol{H}^{1}(Q)$. Since $Q_{c}$ is a (curvilinear) polygon, the scalar potential $\psi_{c}$ belongs to $H^{3 / 2+\varepsilon}\left(\Omega_{c} \backslash \mathcal{V}^{*}\right)$, for any neighborhood $\mathcal{V}^{*}$ of the crack tip and $\varepsilon>0$ small enough. This implies finally that $\gamma_{0} \psi_{c}$ belongs to $H^{1}$ outside the crack mouth $\boldsymbol{x}_{0}$, i.e. $\gamma_{0} \psi_{c} \in H^{1}\left(\Gamma_{0}^{0}\right)$.

Now, consider the problem

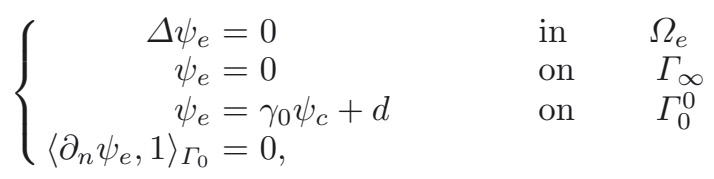

for some $d \in \mathbb{C}$. It follows from Proposition 3.2 that the solution of (4.16) belongs to $H^{1, \alpha}\left(\Omega_{e}\right)$ since the source term $\gamma_{0} \psi_{c}$ is allowed to present a jump at $\boldsymbol{x}_{0}$.

Now, let $\boldsymbol{E}=-i \omega(\boldsymbol{A}+\nabla \psi)$ with $\psi=\psi_{c}$ in $\Omega_{c}$ and $\psi=\psi_{e}$ in $\Omega_{e}$. We easily check that $\boldsymbol{E}$ belongs to $Y_{\alpha}$ and is divergence free in $\Omega_{c}$ and $\Omega_{e}$. In order to prove that $\boldsymbol{E}$ is a solution of (4.2), let $\boldsymbol{v} \in \mathbf{Y}_{\alpha}(\Omega)$ be an arbitrary test field. We deduce from Theorem 3.3 that $\boldsymbol{v}_{c}$ and $\boldsymbol{v}_{e}$ split into a regular and a singular part,

$$
\begin{aligned}
& \boldsymbol{v}_{c}=\boldsymbol{v}_{c, r}+c^{*} \nabla\left(\eta^{*} S^{*}\right)+c^{+} \nabla\left(\eta_{0} S^{\mathrm{Neu}, \omega_{c}^{+}}\right)+c^{-} \nabla\left(\eta_{0} S^{\mathrm{Neu}, \omega_{c}^{-}}\right) \\
& \boldsymbol{v}_{e}=\boldsymbol{v}_{e, r}+c_{0} \nabla\left(\eta_{0} S_{0}\right)+c^{+} S^{+}+c^{-} S^{-}+c_{e} \nabla\left(\eta_{0} S^{\mathrm{Dir}, \omega_{e}}\right) .
\end{aligned}
$$

Let $\boldsymbol{v}_{r}$ the piecewise regular field that coincides with $\boldsymbol{v}_{c, r}$ in $\Omega_{c}$ and with $\boldsymbol{v}_{e, r}$ in $\Omega_{e}$. Notice that curl $\boldsymbol{v}_{r}=$ curl $\boldsymbol{v} \in L^{2}(\Omega)$. Since $\gamma_{n} \boldsymbol{v}_{r, c}=\gamma_{n} \boldsymbol{v}_{c}=0$ simultaneously on $\Sigma$ and on $\Gamma_{0}^{ \pm}$, the trace of $\boldsymbol{v}_{r, c}$ vanishes identically at $\boldsymbol{x}_{0}$ in the sense of $H^{1 / 2}$ which yields $\left[\boldsymbol{v}_{r} \times \boldsymbol{n}\right]_{\mid \Sigma} \equiv 0$ at $\boldsymbol{x}_{0}$. On the other hand, the tangential jump of $\boldsymbol{v}_{r, c}$ is zero on a prolongation of $\Sigma$ into $\Omega_{c}$ and thus $\left[\boldsymbol{v}_{r} \times \boldsymbol{n}\right]_{\mid \Sigma} \equiv 0$ at $\boldsymbol{x}^{*}$. This finally yields $\left[\boldsymbol{v}_{r} \times \boldsymbol{n}\right]_{\mid \Sigma} \in \tilde{H}^{1 / 2}(\Sigma)$ and allows to consider the function $\chi \in H^{1}(\Omega)$, solution of the problem

$$
\begin{aligned}
& \Delta \chi=0 \quad \text { in } \quad \Omega \\
& \begin{aligned}
{[\chi] } & =\left[\boldsymbol{v}_{r} \times \boldsymbol{n}\right] \quad \text { on } & \Sigma \\
\chi & =0 &
\end{aligned}
\end{aligned}
$$

Then, the field $\boldsymbol{v}_{0}=\boldsymbol{v}_{r}-\nabla \chi$ belongs to $\mathcal{H}_{0}(\operatorname{curl} ; Q)$. Now, introduce $\boldsymbol{v}$ in the variational formulation (4.2): we have

$$
\begin{aligned}
a_{R}(\boldsymbol{E}, \boldsymbol{v}) & =\int_{\Omega} \mu^{-1} \operatorname{curl} \boldsymbol{E} \cdot \operatorname{curl} \overline{\boldsymbol{v}} \mathrm{d} \boldsymbol{x}+i \omega \int_{\Omega_{c}} \sigma_{c} \boldsymbol{E} \cdot \overline{\boldsymbol{v}} \mathrm{d} \boldsymbol{x} \\
& =-i \omega \int_{\Omega} \mu^{-1} \operatorname{curl} \boldsymbol{A} \cdot \operatorname{curl} \overline{\boldsymbol{v}_{0}} \mathrm{~d} \boldsymbol{x}+\omega^{2} \int_{\Omega_{c}} \sigma_{c}(\boldsymbol{A}+\nabla \psi) \cdot\left(\overline{\boldsymbol{v}_{0}+\nabla \psi_{c}^{\prime}}\right) \mathrm{d} \boldsymbol{x} \\
& =-i \omega a\left((\boldsymbol{A}, \psi),\left(\boldsymbol{v}_{0}, \psi^{\prime}\right)\right)
\end{aligned}
$$

where $\psi_{c}^{\prime}$ is defined in $\Omega_{c}$ by $\psi_{c}^{\prime}=\chi+c^{*} \eta^{*} S^{*}+c^{+} \eta_{0} S^{\mathrm{Neu}, \omega_{c}^{+}}+c^{-} \eta_{0} S^{\mathrm{Neu}, \omega_{c}^{-}}$. But $(\boldsymbol{A}, \psi)$ is the solution to the $\boldsymbol{A}-\psi$-formulation (4.13) and we thus get, with $\psi_{\mathrm{e}}^{\prime}=\chi+c^{+} S^{+}+c^{-} S^{-}+c_{e} \eta_{0} S^{\mathrm{Dir}, \omega_{e}}$,

$$
a_{R}(\boldsymbol{E}, \boldsymbol{v})=-i \omega \int_{\Omega_{e}} \boldsymbol{J}_{s} \cdot \overline{\boldsymbol{v}_{0}} \mathrm{~d} \boldsymbol{x}=-i \omega \int_{\Omega_{e}} \boldsymbol{J}_{s} \cdot\left(\overline{\boldsymbol{v}-\nabla \psi_{\mathrm{e}}^{\prime}}\right) \mathrm{d} \boldsymbol{x}=-i \omega \int_{\Omega_{e}} \boldsymbol{J}_{s} \cdot \overline{\boldsymbol{v}} \mathrm{d} \boldsymbol{x}
$$

since $\boldsymbol{J}_{s}$ is divergence free in $\Omega_{e}$ and vanishes identically in a vicinity of the boundary $\partial \Omega_{e}$. 
For the converse implication, let $\boldsymbol{E} \in \mathbf{Y}_{\alpha}(\Omega)$ be the solution of (4.2) and built $(\boldsymbol{A}, \psi)$ as follows.

First, consider $\boldsymbol{A}_{0} \in \mathcal{H}_{0}(\operatorname{curl} ; Q) \cap \mathcal{H}(\operatorname{div} ; Q)$, the solution of

$$
\int_{Q}\left(\operatorname{curl} \boldsymbol{A}_{0} \operatorname{curl} \overline{\boldsymbol{B}}+\operatorname{div} \boldsymbol{A}_{0} \operatorname{div} \overline{\boldsymbol{B}}\right) \mathrm{d} \boldsymbol{x}=\frac{i}{\omega} \int_{\Omega} \operatorname{curl} \boldsymbol{E} \operatorname{curl} \overline{\boldsymbol{B}} \mathrm{d} \boldsymbol{x}, \forall \boldsymbol{B} \in \mathcal{H}_{0}(\operatorname{curl} ; Q) \cap \mathcal{H}(\operatorname{div} ; Q) .
$$

Such a solution exists by Lax-Milgram's Lemma since the left-hand side defines a continuous and coercive sesqui-linear form on $\mathcal{H}_{0}(\operatorname{curl} ; Q) \cap \mathcal{H}(\operatorname{div} ; Q)$. By a standard argument, it is clear that $\boldsymbol{A}_{0}$ is divergence free in $Q$ and thus

$$
\operatorname{curl}\left(i \omega \operatorname{curl} \boldsymbol{A}_{0}+\operatorname{curl} \boldsymbol{E}\right)=0 \quad \text { in } \quad \mathcal{D}^{\prime}(\Omega) .
$$

Since in a two-dimensional setting, the vector curl operator is the gradient rotated by $\pi / 2$, we get

$$
i \omega \operatorname{curl} \boldsymbol{A}_{0}+\operatorname{curl} \boldsymbol{E}=i \omega c \quad \text { in } \quad \Omega,
$$

for some constant $c \in \mathbb{C}$.

Next, introduce the vector field

$$
\boldsymbol{F}(\boldsymbol{x})=\frac{1}{2}\left(-x_{2}, x_{1}\right)^{t}
$$

that is obviously divergence free and satisfies $\operatorname{curl} \boldsymbol{F}=1$. Since $Q$ is regular and $\boldsymbol{F} \times \boldsymbol{n} \in H^{1 / 2}\left(\Gamma_{\infty}\right)$, we infer the existence of a scalar function $\theta \in H^{2}(Q)$ such that

$$
\Delta \theta=0 \text { in } Q \text { and } \nabla \theta \times \boldsymbol{n}=\boldsymbol{F} \times \boldsymbol{n} \text { on } \Gamma_{\infty} .
$$

Then, the field $\boldsymbol{D}=\boldsymbol{F}-\nabla \theta$ satisfies

$$
\begin{array}{rll}
\operatorname{div} \boldsymbol{D}=0 & \text { in } & Q, \\
\operatorname{curl} \boldsymbol{D}=1 & \text { in } & Q, \\
\boldsymbol{D} \times \boldsymbol{n}=0 & \text { on } & \Gamma_{\infty},
\end{array}
$$

and we have

$$
\operatorname{curl}\left(i \omega \boldsymbol{A}_{0}+\boldsymbol{E}-i \omega c \boldsymbol{D}\right)=0 \text { in } \Omega .
$$

Since the tangential trace of $i \omega \boldsymbol{A}_{0}+\boldsymbol{E}-i \omega c \boldsymbol{D}$ vanishes on $\Gamma_{\infty}$, the arguments of Section 4.2 apply and yield

$$
i \omega\left(\boldsymbol{A}_{0}-c \boldsymbol{D}\right)+\boldsymbol{E}=-i \omega \nabla \psi \text { in } \Omega,
$$

with

$$
\psi \in\left\{\varphi \in L^{2}(\Omega) \mid \varphi_{c} \in H^{1}\left(\Omega_{c}\right) ; \varphi_{e} \in H^{1, \alpha}\left(\Omega_{e}\right) ; \varphi_{c}-\varphi_{e}=\text { const. on } \Gamma_{0}^{0} ; \varphi=0 \text { on } \Gamma_{\infty}\right\} .
$$

Using the boundary value problem solved by $\boldsymbol{E}$, we obtain that $\left(\boldsymbol{A}, \psi_{c}\right) \in V$ with $\boldsymbol{A}=\boldsymbol{A}_{0}-c \boldsymbol{D}$ is a solution of (4.13).

\section{Numerical RESUlts}

In this section, we aim to provide numerical simulations of eddy currents in a tube with a circular cross section. If the crack is invariant in the direction of the axis of the tube, the computational domain can be reduced to the two-dimensional cross section. The uncracked reference configuration is chosen as in Figure 3 with $\operatorname{diam}\left(\Omega_{c}\right)=1 \mathrm{~mm}$, a lift-off (distance between the conductor and the coil) of $0.2 \mathrm{~mm}$ and a coil of width $0.4 \mathrm{~mm}$. The exterior boundary of $\Omega$ is fixed at a radius $r=1.8 \mathrm{~mm}$. We take $\sigma_{c}=10^{6} \mathrm{~S} / \mathrm{m}$ which corresponds approximatively to the conductivity of stainless steel. The frequency is set to $f=1 \mathrm{MHz}$ with $\omega=2 \pi f$. 


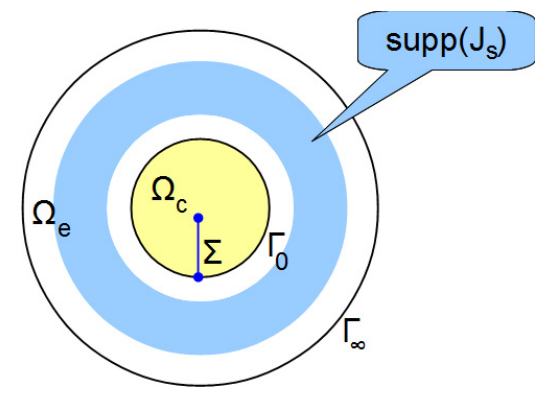

Figure 3. Cross-section of the conductor with encircling coil.

The numerical simulation of the eddy currents will be done with the help of the un-gauged $\boldsymbol{A}-\psi$-formulation. From Theorem 4.2 and Proposition 4.3 we know that there is a unique couple $(\boldsymbol{A}, \psi) \in \boldsymbol{X}^{0}(Q) \times U^{0}\left(\Omega_{c}\right)$ such that

$$
\begin{gathered}
\int_{\Omega} \mu^{-1} \operatorname{curl} \boldsymbol{A} \operatorname{curl} \overline{\boldsymbol{A}^{\prime}} \mathrm{d} \boldsymbol{x}+i \omega \int_{\Omega_{c}} \sigma_{c} \boldsymbol{A} \cdot \overline{\boldsymbol{A}^{\prime}} \mathrm{d} \boldsymbol{x}+i \omega \int_{\Omega_{c}} \sigma_{c} \nabla \psi \cdot \overline{\boldsymbol{A}^{\prime}} \mathrm{d} \boldsymbol{x}=\int_{\Omega_{e}} \boldsymbol{J}_{s} \cdot \overline{\boldsymbol{A}^{\prime}} \mathrm{d} \boldsymbol{x}, \\
\int_{\Omega_{c}} \sigma_{c} \boldsymbol{A} \cdot \nabla \overline{\psi^{\prime}} \mathrm{d} \boldsymbol{x}+\int_{\Omega_{c}} \sigma_{c} \nabla \psi \cdot \nabla \overline{\psi^{\prime}} \mathrm{d} \boldsymbol{x}=0
\end{gathered}
$$

for any $\boldsymbol{A}^{\prime} \in \mathcal{H}_{0}(\operatorname{curl} ; Q)$ and $\psi^{\prime} \in H^{1}\left(\Omega_{c}\right)$.

The discretization of the $\boldsymbol{A}-\psi$-formulation is performed on a conforming mesh of the cracked domain $\Omega$ (see Fig. 4, left picture) by means of edge elements for the discretization of $\boldsymbol{A}$ and first order nodal finite elements for the discretization of $\psi$ in $\Omega_{c}$.

To this end, consider a triangulation $\mathcal{T}_{h}$ of $Q$ that is conforming with the crack geometry, i.e. the intersection of an arbitrary element $K$ of $\mathcal{T}_{h}$ with the crack $\Sigma$ is either a node or an edge of $K$ or the empty set. We denote by $\mathcal{E}_{h}(Q)$ the (oriented) edges of the mesh and by $\mathcal{N}_{h}(Q)$ the nodes of the elements. Since the geometry of our problem is not polygonal, $\mathcal{T}_{h}$ leads to a discrete domain $Q_{h}$ which is different from the original domain $Q$. Notice however that this approximation of the computational domain does not affect the order of the discretization since we use first order elements. In the sequel, we omit the index $h$ in the designation of the different domains. We further assume that the mesh matches approximatively the subdomains $Q_{c}$ and $Q_{e}$.

In order to take into account the discontinuity of the scalar potential across the crack, the nodes situated on the crack are doubled. We denote by $\mathcal{N}\left(\Omega_{c}\right)$ the augmented set of nodes of the conductor $\Omega_{c}$.

The discretization space for the vector potential $\boldsymbol{A}$ is defined by

$$
\boldsymbol{X}_{h}(Q)=\left\{\boldsymbol{A}_{h} \in \mathcal{H}_{0}(\operatorname{curl} ; Q) \mid \boldsymbol{A}_{h \mid K} \in \mathcal{R}_{K} \forall K \in \mathcal{T}_{h}\right\} .
$$

Here,

$$
\mathcal{R}_{K}=\left\{\boldsymbol{a}+b\left(\begin{array}{c}
x_{2} \\
-x_{1}
\end{array}\right) \mid \boldsymbol{a} \in \mathbb{R}^{2}, b \in \mathbb{R}\right\}
$$

is the local approximation space for two-dimensional edge elements. For any edge $e \in \mathcal{E}_{h}(Q)$, we introduce the linear form

$$
\ell_{e}(\boldsymbol{v})=\int_{e} \boldsymbol{v} \cdot \boldsymbol{t}_{e} \mathrm{~d} s
$$

where $\boldsymbol{t}_{e}$ is the unit tangent vector in the direction of the edge $e$. The associated basis function $\boldsymbol{w}_{e}$ is defined in the usual way by

$$
\begin{aligned}
& \boldsymbol{w}_{e \mid K} \in \mathcal{R}_{K} \forall K \in \mathcal{T}_{h}, \\
& \ell_{\mathrm{e}^{\prime}}\left(\boldsymbol{w}_{e}\right)=\delta_{e e^{\prime}} \forall \mathrm{e}^{\prime} \in \mathcal{E}_{h}(Q) .
\end{aligned}
$$


Then, $\boldsymbol{X}_{h}(Q)$ is spanned by the basis functions $\boldsymbol{w}_{e}$ corresponding to interior edges $e \in \mathcal{E}_{h}(Q) \backslash \Gamma_{\infty}$ and we note $N_{E}$ the dimension of $\boldsymbol{X}_{h}(Q)$.

Similarly, we introduce P1 Lagrange finite elements for the discretization of the scalar potential $\psi$ which is defined on the conductor $\Omega_{c}$ only:

$$
U_{h}\left(\Omega_{c}\right)=\left\{\psi_{h} \in H^{1}\left(\Omega_{c}\right) \mid \psi_{h \mid K} \in \mathbb{P}_{1}(K) \forall K \in \mathcal{T}_{h} \cap \Omega_{c}\right\} .
$$

$U_{h}\left(\Omega_{c}\right)$ is spanned by the nodal basis functions $w_{I}$ corresponding to the augmented nodes of $\mathcal{N}\left(\Omega_{c}\right)$ situated in $\Omega_{c}$. We denote by $N_{c}=\operatorname{card}\left(\mathcal{N}\left(\Omega_{c}\right)\right)$ the dimension of $U_{h}\left(\Omega_{c}\right)$.

As for the continuous problem, the discrete problem is well posed under appropriate gauge conditions only. To this end, we introduce the space of P1 Lagrange finite elements on the whole computational domain:

$$
S_{h}(Q)=\left\{\xi_{h} \in H_{0}^{1}(Q) \mid \xi_{h \mid K} \in \mathbb{P}_{1}(K) \forall K \in \mathcal{T}_{h}\right\} .
$$

Then, the space of discrete divergence free vector fields is given by

$$
\boldsymbol{X}_{h}^{0}(Q)=\left\{\boldsymbol{v}_{h} \in \boldsymbol{X}_{h} \mid\left(\boldsymbol{v}_{h}, \nabla \xi_{h}\right)=0 \forall \xi_{h} \in S_{h}(Q)\right\} .
$$

Finally, the space of gauged scalar potentials is defined by

$$
U_{h}^{0}\left(\Omega_{c}\right)=U_{h}\left(\Omega_{c}\right) \cap L_{0}^{2}\left(\Omega_{c}\right) .
$$

The discrete gauged problem given on the space $V_{h}=\boldsymbol{X}_{h}^{0}(Q) \times U_{h}^{0}\left(\Omega_{c}\right)$ reads as follows

$$
\left\{\begin{array}{l}
\text { Find }\left(\boldsymbol{A}_{h}, \psi_{h}\right) \in V_{h} \text { such that } \\
a\left(\left(\boldsymbol{A}_{h}, \psi_{h}\right),\left(\boldsymbol{A}_{h}^{\prime}, \psi_{h}^{\prime}\right)\right)=\ell\left(\left(\boldsymbol{A}_{h}^{\prime}, \psi_{h}^{\prime}\right)\right) \forall\left(\boldsymbol{A}_{h}^{\prime}, \psi_{h}^{\prime}\right) \in V_{h} .
\end{array}\right.
$$

Problem (5.1) admits a unique solution according to Theorem 2.4 in [21] which still holds in our setting of a cracked domain since $\boldsymbol{A}_{h}$ is sought in a discretization space that is $\mathcal{H}($ curl)-conforming for the uncracked domain.

The vector spaces $\boldsymbol{X}_{h}^{0}(Q)$ and $U_{h}^{0}\left(\Omega_{c}\right)$ of gauged potentials are not suited for the implementation of the discrete problem. Different discrete gauge conditions have been studied in [2]. They result however in poorly conditioned linear systems and a high number of conjugate gradient iterations (see [35] for a discussion of this item). Another way to deal with the gauge condition is to consider a regularized formulation of the $\boldsymbol{A}-\psi$ formulation by adding a penalization term $\left(\operatorname{div} \boldsymbol{A}, \operatorname{div} \boldsymbol{A}^{\prime}\right)$ to the curl-curl-term in (4.12). The new formulation is then defined for vector potentials in $\mathcal{H}_{0}(\operatorname{curl} ; Q) \cap \mathcal{H}(\operatorname{div} ; Q)$. For the time-harmonic Maxwell equations, this technique has been extensively studied (see e.g. $[6,10,17,28]$ ). For the eddy current problem, the regularized formulation has been analyzed in [1]. If the domain $Q$ is regular or Lipschitz and convex, such a formulation allows to use standard Lagrange finite elements. In the case of a non-convex polygon, however, discretization by means of such nodal finite elements does not lead to a converging method unless the singularities are explicitly added to the discretization space or taken into account via a weighted regularization. In the present paper, we prefer to use edge elements for the vector potential and Lagrange finite elements for the scalar potential. This allows to compute the total electric field $\boldsymbol{E}$ in an easy way, since the gradients of P1 Lagrange elements are contained in the space of edge elements. In order to take into account the divergence constraint, we use the un-gauged $\boldsymbol{A}-\psi$-formulation together with an appropriate implementation of the right hand side. This has been proposed in [36] for a magnetostatic problem. To this end, the source term $\boldsymbol{J}_{\boldsymbol{s}}$ is actually implemented as the (vector) curl of a scalar potential $T_{0}, \boldsymbol{J}_{s}=\operatorname{curl} T_{0}$. The existence of $T_{0}$ follows from div $\boldsymbol{J}_{s}=0$. We then have by integration by parts

$$
\int_{\Omega_{e}} \boldsymbol{J}_{s} \cdot \overline{\boldsymbol{A}_{h}^{\prime}} \mathrm{d} \boldsymbol{x}=\int_{Q} T_{0} \operatorname{curl} \overline{\boldsymbol{A}_{h}^{\prime}} \mathrm{d} \boldsymbol{x}
$$


since $\boldsymbol{A}_{h}^{\prime}$ belongs to $\mathcal{H}_{0}($ curl; $Q)$. The un-gauged discrete problem now reads

$$
\left\{\begin{array}{l}
\text { Find }\left(\boldsymbol{A}_{h}, \psi_{h}\right) \in \boldsymbol{X}_{h}(Q) \times U_{h}\left(\Omega_{c}\right) \text { such that } \\
a\left(\left(\boldsymbol{A}_{h}, \psi_{h}\right),\left(\boldsymbol{A}_{h}^{\prime}, \psi_{h}^{\prime}\right)\right)=\ell_{h}\left(\left(\boldsymbol{A}_{h}^{\prime}, \psi_{h}^{\prime}\right)\right) \forall\left(\boldsymbol{A}_{h}^{\prime}, \psi_{h}^{\prime}\right) \in \boldsymbol{X}_{h}(Q) \times U_{h}\left(\Omega_{c}\right),
\end{array}\right.
$$

where

$$
\ell_{h}\left(\left(\boldsymbol{A}_{h}, \psi_{h}\right)\right)=\int_{Q} T_{0} \operatorname{curl} \overline{\boldsymbol{A}_{h}^{\prime}} \mathrm{d} \boldsymbol{x} .
$$

Actually, we are able to prove that the solution $\left(\boldsymbol{A}_{h}, \psi_{h}\right)$ of problem (5.1) satisfies the variational equation for any field $\left(\boldsymbol{A}_{h}^{\prime}, \psi_{h}^{\prime}\right)$ in the un-gauged space $\boldsymbol{X}_{h}(Q) \times U_{h}\left(\Omega_{c}\right)$ :

Proposition 5.1. Let $\left(\boldsymbol{A}_{h}, \psi_{h}\right) \in V_{h}$ be the unique solution of (5.1) with right hand side $\ell_{h}(\cdot)$. Then

$$
a\left(\left(\boldsymbol{A}_{h}, \psi_{h}\right),\left(\boldsymbol{A}_{h}^{\prime}, \psi_{h}^{\prime}\right)\right)=\ell_{h}\left(\left(\boldsymbol{A}_{h}^{\prime}, \psi_{h}^{\prime}\right)\right) \forall\left(\boldsymbol{A}_{h}^{\prime}, \psi_{h}^{\prime}\right) \in \boldsymbol{X}_{h}(Q) \times U_{h}\left(\Omega_{c}\right) .
$$

Proof. First notice that the scalar potential acts in the variational formulation only through its gradient. Therefore, let $\psi_{h}^{\prime} \in U_{h}\left(\Omega_{c}\right)$ and introduce the function

$$
\tilde{\psi}_{h}^{\prime}=\psi_{h}^{\prime}-\frac{1}{\operatorname{meas}\left(\Omega_{c}\right)} \int_{\Omega_{c}} \psi_{h}^{\prime} \mathrm{d} \boldsymbol{x} .
$$

The function $\tilde{\psi}_{h}^{\prime}$ has zero mean value and belongs to the gauged vector space $U_{h}^{0}\left(\Omega_{c}\right)$. Since $\nabla \tilde{\psi}_{h}^{\prime}=\nabla \psi_{h}^{\prime}$ and $\ell_{h}(\cdot)$ does not depend on $\psi_{h}^{\prime}$, the variational equality holds for any $\psi_{h}^{\prime} \in U_{h}\left(\Omega_{c}\right)$.

Next, let $\boldsymbol{A}_{h}^{\prime}$ belong to $\boldsymbol{X}_{h}(Q)$. According to the definition of $\boldsymbol{X}_{h}^{0}(Q)$, we decompose $\boldsymbol{A}_{h}^{\prime}$ into

$$
\boldsymbol{A}_{h}^{\prime}=\tilde{\boldsymbol{A}}_{h}^{\prime}+\nabla \tau_{h}^{\prime}
$$

with $\tilde{\boldsymbol{A}}_{h}^{\prime} \in \boldsymbol{X}_{h}^{0}(Q)$ and $\tau_{h}^{\prime} \in S_{h}(Q)$. We thus have curl $\boldsymbol{A}_{h}^{\prime}=$ curl $\tilde{\boldsymbol{A}}_{h}^{\prime}$ which yields

$$
a\left(\left(\boldsymbol{A}_{h}, \psi_{h}\right),\left(\boldsymbol{A}_{h}^{\prime}, \psi_{h}^{\prime}\right)\right)=\int_{\Omega} \mu^{-1} \operatorname{curl} \boldsymbol{A}_{h} \overline{\operatorname{curl} \tilde{\boldsymbol{A}}_{h}^{\prime}} \mathrm{d} \boldsymbol{x}+i \omega \int_{\Omega_{c}} \sigma_{c}\left(\boldsymbol{A}_{h}+\nabla \psi_{h}\right) \cdot \overline{\left(\tilde{\boldsymbol{A}}_{h}^{\prime}+\nabla\left(\tau_{h}^{\prime}+\psi_{h}^{\prime}\right)\right)} \mathrm{d} \boldsymbol{x} .
$$

The function $\tau_{h \mid \Omega_{c}}^{\prime}+\psi_{h}^{\prime}$ belongs to $U_{h}\left(\Omega_{c}\right)$ and is admissible as a scalar potential of the test field. We thus deduce from (5.1) that

$$
a\left(\left(\boldsymbol{A}_{h}, \psi_{h}\right),\left(\boldsymbol{A}_{h}^{\prime}, \psi_{h}^{\prime}\right)\right)=\ell_{h}\left(\left(\tilde{\boldsymbol{A}}_{h}^{\prime}, \tau_{h}^{\prime}+\psi_{h}^{\prime}\right)\right)=\int_{Q} T_{0} \overline{\operatorname{curl} \tilde{\boldsymbol{A}}_{h}^{\prime}} \mathrm{d} \boldsymbol{x}=\int_{Q} T_{0} \overline{\operatorname{curl} \boldsymbol{A}_{h}^{\prime}} \mathrm{d} \boldsymbol{x}=\ell_{h}\left(\left(\boldsymbol{A}_{h}^{\prime}, \psi_{h}^{\prime}\right)\right)
$$

since $\tilde{\boldsymbol{A}}_{h}^{\prime}=\boldsymbol{A}_{h}^{\prime}-\nabla \tau_{h}^{\prime}$.

Notice that the implementation of the right hand side is primordial in order to get the result of Proposition 5.1. Indeed, consider the original right hand side of the problem

$$
\ell\left(\left(\boldsymbol{A}_{h}^{\prime}, \psi_{h}^{\prime}\right)\right)=\int_{Q} \boldsymbol{J}_{s} \cdot \overline{\boldsymbol{A}_{h}^{\prime}} \mathrm{d} \boldsymbol{x} .
$$

Due to numerical integration in the implementation of $\boldsymbol{J}_{s}$, we have in general

$$
\int_{Q} \boldsymbol{J}_{s} \cdot \nabla \overline{\tau_{h}^{\prime}} \mathrm{d} \boldsymbol{x} \approx \int_{Q} \boldsymbol{J}_{s, h} \cdot \nabla \overline{\tau_{h}^{\prime}} \mathrm{d} \boldsymbol{x} \neq 0
$$

since the approximated source field will not be exactly divergence free. Consequently, the right hand side of the resulting linear system $\mathbb{A} X=F$ will not be exactly in the range of the matrix $\mathbb{A}$. Iterative resolution methods 

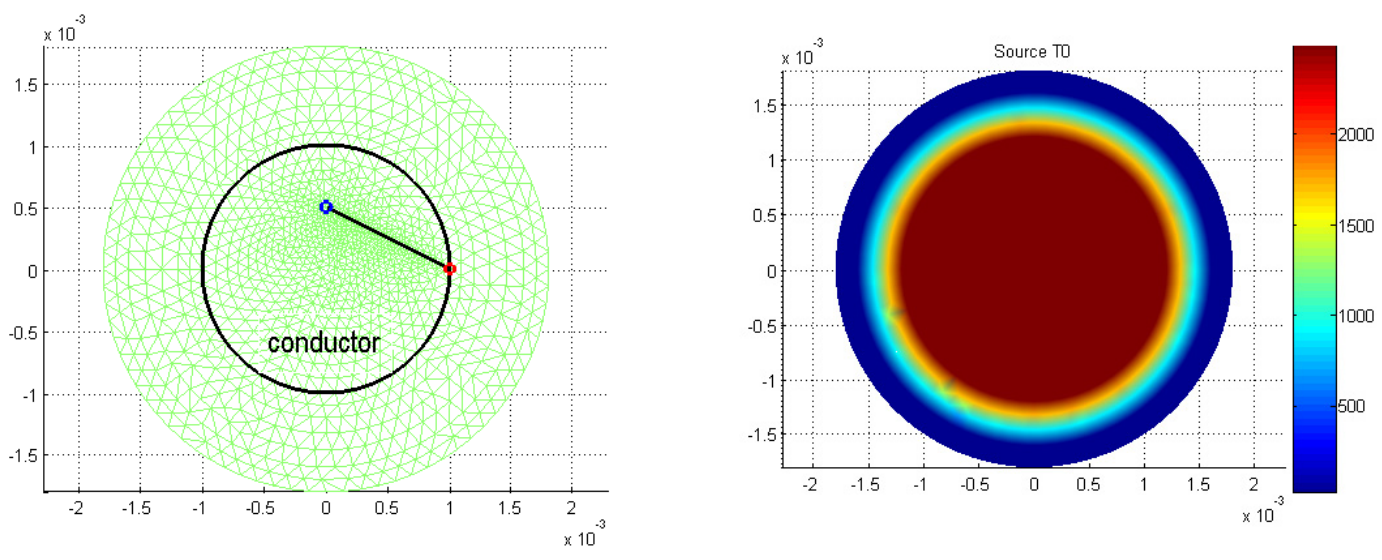

Figure 4. Mesh (left) and source field $T_{0}$ (right).

will then stabilize around a constant residual (see [36] for a similar study of the magnetostatic problem). Following [36], we overcome this difficulty by using the potential $T_{0}$ in the linear form $\ell_{h}(\cdot)$. Here, we obviously get

$$
\int_{Q} T_{0} \operatorname{curl} \nabla \overline{\tau_{h}^{\prime}} \mathrm{d} \boldsymbol{x}=\int_{Q} T_{0, h} \operatorname{curl} \nabla \overline{\tau_{h}^{\prime}} \mathrm{d} \boldsymbol{x}=0
$$

for any approximation $T_{0, h}$ of the potential $T_{0}$. This formulation ensures that the right hand side satisfies the necessary compatibility conditions and belongs to the range of the matrix. A conjugate gradient algorithm may then be applied to solve the linear system numerically. Indeed, starting from an arbitrary vector $X^{(0)}$, the residual $r^{(k)}=F-\mathbb{A} X^{(k)}$ at iteration $k$ belongs to the range of the matrix $\mathbb{A}$ whenever the right hand side $F$ does. Hence, the conjugate gradient algorithm is well defined and converges under the usual conditions. Notice however that the matrix $\mathbb{A}$ is complex symmetric but not hermitian. Conjugate gradient-type methods have been developed for such kind of linear systems in [22].

In our application, $T_{0}$ is given analytically as a function of the radius $r$ ( $c f$. Fig. 4 , right picture):

$$
T_{0}(r)= \begin{cases}I & \text { if } \quad r \leq r_{1}, \\ I \frac{r_{2}-r}{r_{2}-r_{1}} & \text { if } \quad r_{1} \leq r \leq r_{2}, \\ 0 & \text { otherwise }\end{cases}
$$

Notice that the support of the source term $\boldsymbol{J}_{s}$ is the ring between $r_{1}$ and $r_{2}$. Therefore, we assume that $2 r_{1}>\operatorname{diam}\left(\Omega_{c}\right)$ such that $\operatorname{supp}\left(\boldsymbol{J}_{s}\right)$ does not meet the conducting region $\Omega_{c}$.

In Figures 5 and 6, we represent the real and imaginary part of the eddy currents $\boldsymbol{J}=\sigma \boldsymbol{E}$ in the conductor. We clearly see that the currents are concentrated near the surface $\Gamma$ of the conductor and the crack with an orientation tangential to $\Gamma_{0}$ and the crack, illustrating the skin effect of eddy currents. In Figure 7 , we represent the amplitude $|J|$ in the total domain as well as near the crack. The right picture of Figure 7 exhibits the singularity at the crack tip.

As for the behavior near the crack mouth, notice first of all that $\Omega_{c}^{ \pm}$is convex near $\boldsymbol{x}_{0}$. Consequently, no singularity occurs inside the conductor. On the other hand, the exterior domain $\Omega_{e}$ is regular and the electric field $\boldsymbol{E}_{e}$ thus behaves as $\nabla S_{0}$ near the crack mouth. According to Proposition 3.2, the singular coefficient of the singular function $S_{0}$ is given by the potential drop $c_{0}=\psi_{c}^{+}\left(\boldsymbol{x}_{0}\right)-\psi_{c}^{-}\left(\boldsymbol{x}_{0}\right)$ and takes a value of $\left|c_{0}\right| \approx 1 e-10$ in the present simulation. Notice that the order of magnitude of the electric field inside the conductor is of $1 e-7\left[\mathrm{~V} \mathrm{~m}^{-1}\right]$. In order to get an idea of the asymptotic behavior near $\boldsymbol{x}_{0}$ outside the conductor, we represent 

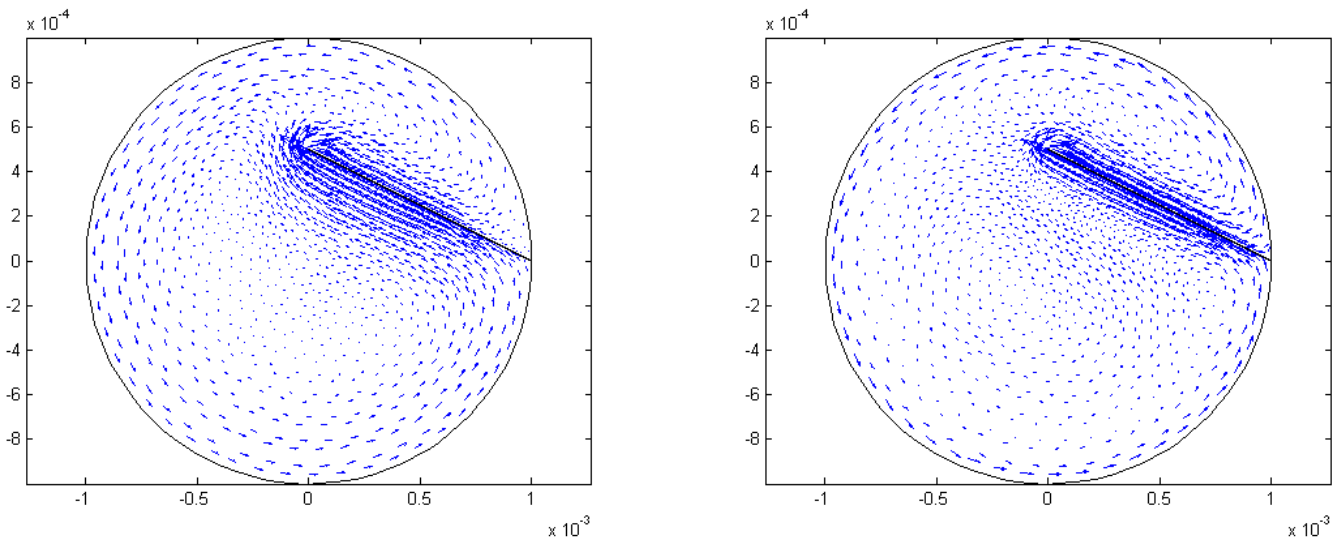

FiguRE 5. Orientation of eddy currents in the conductor: real (left) and imaginary (right) part.
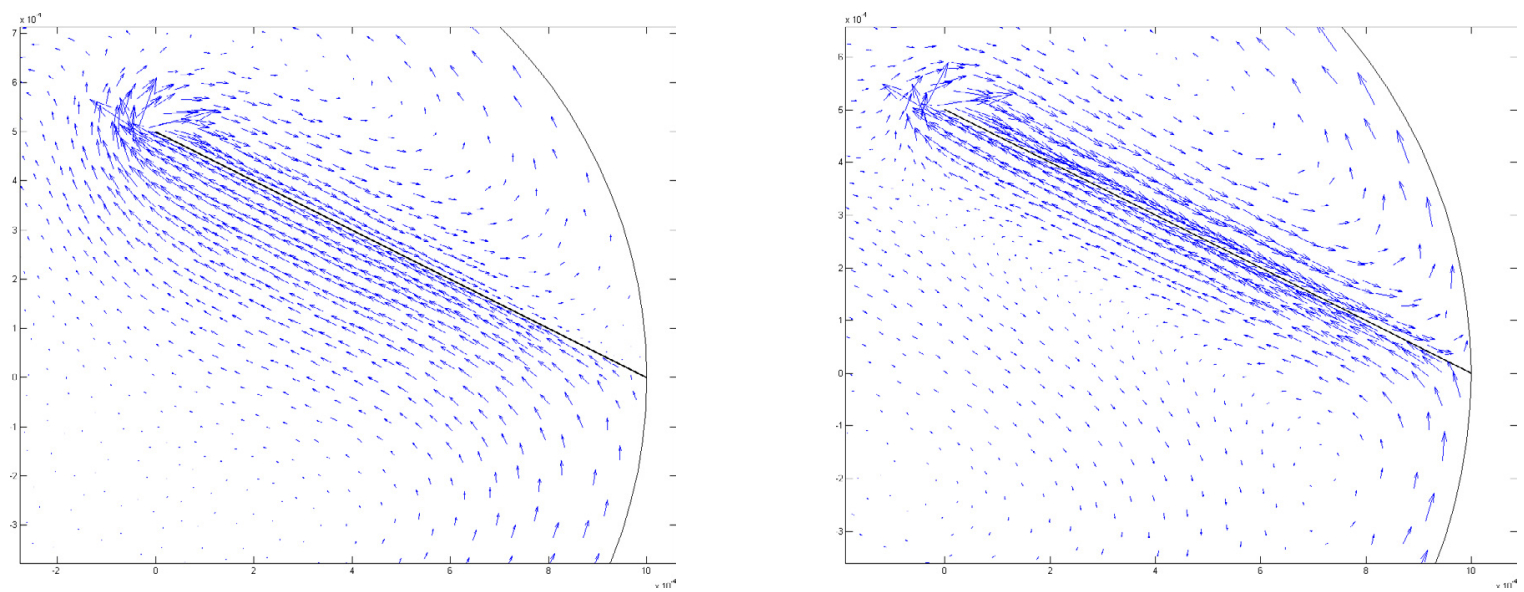

FiguRE 6. Eddy currents near the crack: real (left) and imaginary (right) part.
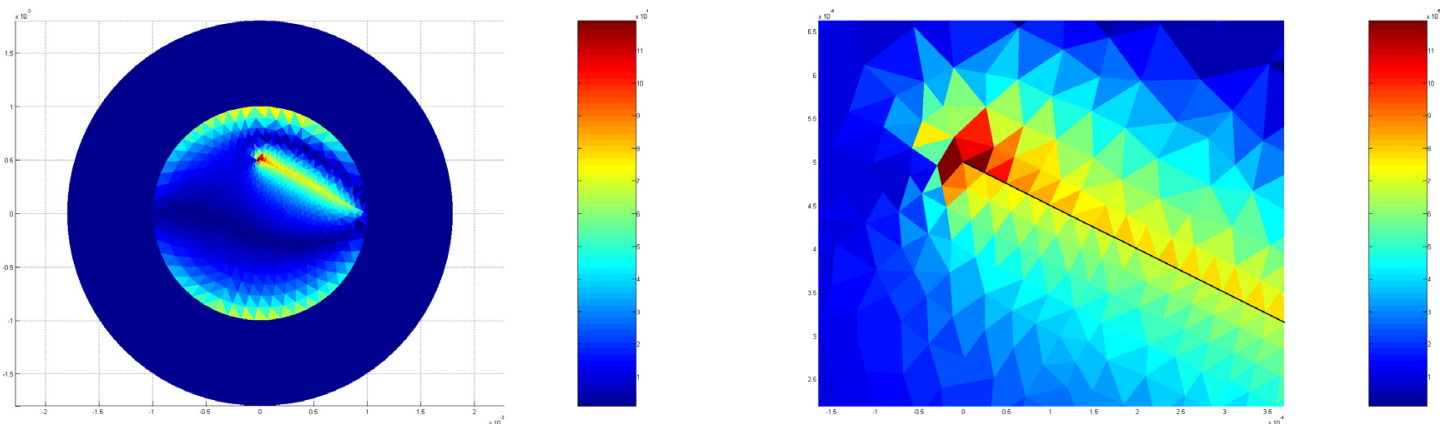

Figure 7. Amplitude $|J|$ of the eddy currents. 

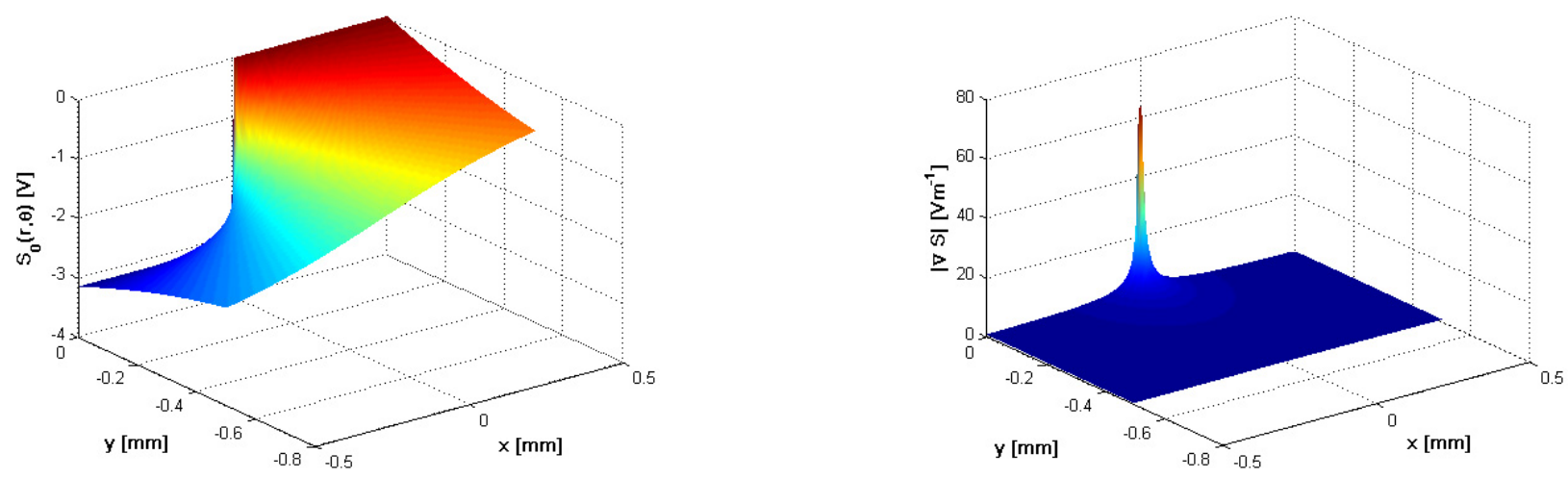

Figure 8. Behavior of the singular function $S_{0}$ (left) and its gradient (right) near the crack mouth.

in Figure 8 the singular potential $S_{0}$ (left) and the amplitude $\left|\nabla S_{0}\right|$ (right) in a subdomain of the half-space $\left\{x_{2}<0\right\}$.

According to the proof of Proposition 3.2 and Theorem 4.4, it is possible to compute the electric field from the solution $\left(\boldsymbol{A}, \psi_{c}\right)$ of the $\boldsymbol{A}-\psi$-formulation through the following algorithm:

1. Compute the potential gap $c_{0}=\psi_{c}^{+}\left(\boldsymbol{x}_{0}\right)-\psi_{c}^{-}\left(\boldsymbol{x}_{0}\right)$.

2. Solve the following scalar problem

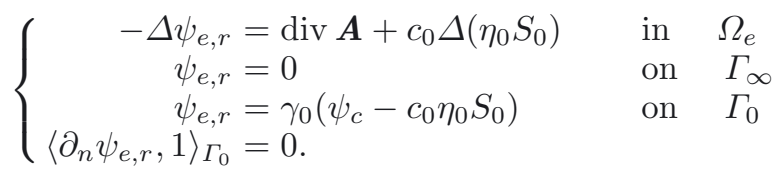

The discretization of the above problem can be performed by standard Lagrange finite elements.

3. Set $\psi_{e}=\psi_{e, r}+c_{0} \eta_{0} S_{0} \in H^{1, \alpha}\left(\Omega_{e}\right)$.

4. Set $\boldsymbol{E}_{e}=-i \omega\left(\boldsymbol{A}+\nabla \psi_{e}\right)$.

\section{Appendix A. Resolution of LAplace's EQUATion in AN open SECTOR WITH DISCONTINUOUS BOUNDARY DATA}

In this appendix, we address the resolution of a special boundary value problem with discontinuous data. A detailed presentation of such kind of problems can be found in [34].

Consider the infinite open sector of angle $\omega$ in $\mathbb{R}^{2}$,

$$
\mathcal{S}_{\omega}=\{(r \cos \theta, r \sin \theta) \mid r>0,0<\theta<\omega\} .
$$

The boundary of $\mathcal{S}_{\omega}$ splits into two rays, $\Gamma^{+}$and $\Gamma^{-}$, corresponding to $\theta=0$ and $\theta=\omega$, respectively.

Proposition A.1. Let $f$ be given by

$$
f(x)=\left\{\begin{array}{lll}
0 & \text { on } & \Gamma^{+} \\
1 & \text { on } & \Gamma^{-} .
\end{array}\right.
$$

Then a solution to the boundary-value problem

$$
\left\{\begin{array}{rll}
\Delta u=0 & \text { in } & \mathcal{S}_{\omega} \\
u=f & \text { on } & \partial \mathcal{S}_{\omega}
\end{array}\right.
$$


is given by

$$
S_{0}(r, \theta)=\frac{\theta}{\omega}
$$

The function $S_{0}$ belongs to the weighted Sobolev space $H^{1, \alpha}(\mathcal{V}(\mathbf{0}))$ for any $\alpha>0$ and any bounded neighborhood $\mathcal{V}(\mathbf{0})$ in $\mathcal{S}_{\omega}$.

Proof. It can be proved by a direct computation that the function $S_{0}$ defined by (A.2) is a solution of the boundary value problem (A.1).

In order to exhibit the regularity of the solution $S_{0}$, notice that for any disc $B_{R}(\mathbf{0})$ centered at the origin, we have

$$
\left\|r^{\alpha-1} S_{0}\right\|_{0, B_{r}(\mathbf{0}) \cap \mathcal{S}_{\omega}}^{2}=\int_{0}^{R} \int_{0}^{\omega} r^{2 \alpha-2}\left|S_{0}(r, \theta)\right|^{2} r \mathrm{~d} \theta \mathrm{d} r=\frac{1}{\omega^{2}} \int_{0}^{R} \int_{0}^{\pi}|\theta|^{2} r^{2 \alpha-1} \mathrm{~d} \theta \mathrm{d} r<\infty
$$

if $\alpha>0$. In the same way, we get

$$
\left\|r^{\alpha} \nabla S_{0}\right\|_{0, B_{r}(\mathbf{0}) \cap \mathcal{S}_{\omega}}^{2}=\frac{1}{\omega^{2}} \int_{0}^{R} \int_{0}^{\pi} r^{2 \alpha-1} \mathrm{~d} \theta \mathrm{d} r<\infty
$$

which proves that $S_{0}$ belongs locally to the weighted Sobolev space $H^{1, \alpha}$ near $\mathbf{0}$.

\section{Appendix B. Vector and scalar potentials in $L^{p}$}

In this section, we adapt several results from [5] for our purposes. Let us introduce the following sub-spaces of $\boldsymbol{L}^{p}\left(\Omega_{e}\right)$ :

$$
\begin{aligned}
& H^{p}\left(\operatorname{div} ; \Omega_{e}\right)=\left\{\boldsymbol{v} \in \boldsymbol{L}^{p}\left(\Omega_{e}\right) \mid \operatorname{div} \boldsymbol{v} \in L^{p}\left(\Omega_{e}\right)\right\}, \\
& H^{p}\left(\operatorname{curl} ; \Omega_{e}\right)=\left\{\boldsymbol{v} \in L^{p}\left(\Omega_{e}\right) \mid \operatorname{curl} \boldsymbol{v} \in L^{p}\left(\Omega_{e}\right)\right\} .
\end{aligned}
$$

On $H^{p}\left(\operatorname{div} ; \Omega_{e}\right)$, the normal trace $\gamma_{n} \boldsymbol{v}=\boldsymbol{v} \cdot \boldsymbol{n}$ is defined as an element of the dual space of $W^{1-1 / p^{\prime}, p^{\prime}}\left(\partial \Omega_{e}\right)$ where $\frac{1}{p}+\frac{1}{p^{\prime}}=1$. Similarly, on $H^{p}\left(\operatorname{curl} ; \Omega_{e}\right)$, the tangential trace $\gamma_{t} \boldsymbol{v}=\boldsymbol{v} \times \boldsymbol{n}$ belongs to $\left(W^{1-1 / p^{\prime}, p^{\prime}}\left(\partial \Omega_{e}\right)\right)^{\prime}$. We thus introduce the spaces

$$
\begin{aligned}
& H_{0, \Gamma}^{p}\left(\operatorname{div} ; \Omega_{e}\right)=\left\{\boldsymbol{v} \in H^{p}\left(\operatorname{div} ; \Omega_{e}\right) \mid \gamma_{n} \boldsymbol{v}=0 \text { on } \Gamma\right\} \\
& H_{0, \Gamma_{\infty}}^{p}\left(\operatorname{curl} ; \Omega_{e}\right)=\left\{\boldsymbol{v} \in H^{p}\left(\operatorname{curl} ; \Omega_{e}\right) \mid \gamma_{t} \boldsymbol{v}=0 \text { on } \Gamma_{\infty}\right\}
\end{aligned}
$$

The next theorem follows from Theorem 3.1 in [5].

Theorem B.1. A vector field $\boldsymbol{v} \in \boldsymbol{L}^{p}\left(\Omega_{e}\right)$ satisfies

$$
\operatorname{div} \boldsymbol{v}=0 \text { in } \Omega_{e} \text { and } \gamma_{n} \boldsymbol{v}=0 \text { on } \Gamma_{0}
$$

if and only if there is a stream function $\psi \in W^{1, p}\left(\Omega_{e}\right)$ such that

$$
\boldsymbol{v}=\operatorname{curl} \psi \text { in } \Omega_{e} \text {, and } \psi=0 \text { on } \Gamma_{0} \text {. }
$$

The vector field $\psi$ is uniquely determined and satisfies the estimate

$$
\|\psi\|_{W^{1, p}\left(\Omega_{e}\right)} \lesssim\|\boldsymbol{u}\|_{L^{p}\left(\Omega_{e}\right)} .
$$


Proof. Let $\boldsymbol{v} \in \boldsymbol{L}^{p}\left(\Omega_{e}\right)$ be as in (B.5). We obviously have $\left\langle\gamma_{n} \boldsymbol{v}, 1\right\rangle_{\Gamma_{0}}=0$. Further, we have

$$
\left\langle\gamma_{n} \boldsymbol{v}, 1\right\rangle_{\partial \Omega_{e}}=\int_{\Omega_{e}} \operatorname{div} \boldsymbol{v} \mathrm{d} \boldsymbol{x}=0,
$$

and thus

$$
\left\langle\gamma_{n} \boldsymbol{v}, 1\right\rangle_{\Gamma_{\infty}}=-\left\langle\gamma_{n} \boldsymbol{v}, 1\right\rangle_{\Gamma_{0}}=0 .
$$

Therefore, according to Theorem 3.1. in [5] in its two-dimensional version, there is a stream function $\psi_{0} \in$ $W^{1, p}\left(\Omega_{e}\right)$ such that

$$
\boldsymbol{v}=\operatorname{curl} \psi_{0} \text { in } \Omega_{e}
$$

Since $\gamma_{n} \boldsymbol{v}=0$ on $\Gamma_{0}$, we have

$$
\partial_{s} \psi_{0}=\gamma_{n} \operatorname{curl} \psi_{0}=0 \text { on } \Gamma_{0}
$$

which yields $\psi_{0}=c \in \mathbb{C}$ on $\Gamma_{0}$. Finally, we let $\psi=\psi_{0}-c$ in order to get a stream function satisfying (B.6). From Theorem 3.1 in [5], we get the estimate

$$
\left\|\psi_{0}\right\|_{W^{1, p}\left(\Omega_{e}\right)} \lesssim\|\boldsymbol{u}\|_{L^{p}\left(\Omega_{e}\right)}
$$

But $\psi$ belongs to $W_{0, \Gamma_{0}}^{1, p}\left(\Omega_{e}\right)$ and Poincaré's inequality thus yields

$$
\|\psi\|_{L^{p}\left(\Omega_{e}\right)} \lesssim\|\nabla \psi\|_{L^{p}\left(\Omega_{e}\right)}=\left\|\nabla \psi_{0}\right\|_{L^{p}\left(\Omega_{e}\right)}
$$

which yields (B.7).

Theorem B.2. Let $\boldsymbol{f} \in \boldsymbol{L}^{p}\left(\Omega_{e}\right)$ such that $\operatorname{curl} \boldsymbol{f}=0$ in $\Omega_{e}$ and $\gamma_{t} \boldsymbol{f}=0$ on $\Gamma_{\infty}$. There is a unique function $\varphi \in W_{0, \Gamma_{\infty}}^{1, p}\left(\Omega_{e}\right)$ such that

$$
f=\nabla \varphi
$$

Proof. Consider the gradient as a linear operator from $\mathcal{D}(\operatorname{grad})=W_{0, \Gamma_{\infty}}^{1, p}\left(\Omega_{e}\right) \subset L^{p}\left(\Omega_{e}\right)$ into $\boldsymbol{L}^{p}\left(\Omega_{e}\right)$. Then the divergence operator is the adjoint of grad with

$$
\operatorname{div}: \mathcal{D}(\operatorname{div})=H_{0, \Gamma}^{p^{\prime}}\left(\operatorname{div} ; \Omega_{e}\right) \subset \boldsymbol{L}^{p^{\prime}}\left(\Omega_{e}\right) \rightarrow L^{p^{\prime}}\left(\Omega_{e}\right) .
$$

Indeed, for $(\varphi, v) \in \mathcal{D}(\operatorname{grad}) \times \mathcal{D}($ div $)$, we have

$$
\int_{\Omega_{e}} \boldsymbol{v} \cdot \nabla \varphi \mathrm{d} \boldsymbol{x}=-\int_{\Omega_{e}} \operatorname{div} \boldsymbol{v} \varphi \mathrm{d} \boldsymbol{x}
$$

since $\gamma_{n} \boldsymbol{v}=0$ on $\Gamma$ and $\varphi=0$ on $\Gamma_{\infty}$. With the help of Poincaré's inequality, we can show that the range of $\operatorname{grad}$ is closed in $\boldsymbol{L}^{p}\left(\Omega_{e}\right)$. We thus have

$$
\mathcal{R}(\operatorname{grad})=\operatorname{Ker}(\operatorname{div})^{\perp}=\left\{\boldsymbol{f} \in \boldsymbol{L}^{p}\left(\Omega_{e}\right) \mid \int_{\Omega_{e}} \boldsymbol{f} \cdot \boldsymbol{v}=0 \forall \boldsymbol{v} \in H_{0, \Gamma}^{p^{\prime}}\left(\operatorname{div} ; \Omega_{e}\right): \operatorname{div} \boldsymbol{v}=0 \text { in } \Omega_{e}\right\}
$$

Now, let $\boldsymbol{f} \in \boldsymbol{L}^{p}\left(\Omega_{e}\right)$ such that $\operatorname{curl} \boldsymbol{f}=0$ in $\Omega_{e}$ and $\gamma_{t} \boldsymbol{f}=0$ on $\Gamma_{\infty}$. We have to evaluate

$$
\int_{\Omega_{e}} f \cdot \boldsymbol{v} \mathrm{d} \boldsymbol{x}
$$

for an arbitrary vector field $\boldsymbol{v} \in \operatorname{Ker}(\operatorname{div})$. Thus, consider $\boldsymbol{v} \in H_{0, \Gamma}^{p^{\prime}}\left(\operatorname{div} ; \Omega_{e}\right)$ such that $\operatorname{div} \boldsymbol{v}=0$ in $\Omega_{e}$. According to Theorem B.1, there is a stream function $\psi \in W^{1, p^{\prime}}\left(\Omega_{e}\right)$ such that $\boldsymbol{v}_{0}=\operatorname{curl} \psi$ and $\psi=0$ on $\Gamma_{0}$. It thus follows by integration by parts and the boundary conditions satisfied by $\psi$ and $\boldsymbol{f}$ that

$$
\int_{\Omega_{e}} \boldsymbol{f} \cdot \boldsymbol{v} \mathrm{d} \boldsymbol{x}=\left\langle\gamma_{t} \boldsymbol{f}, \psi\right\rangle_{\Gamma_{\infty}}+\left\langle\gamma_{t} \boldsymbol{f}, \psi\right\rangle_{\Gamma_{0}}=0
$$

Hence, $f$ belongs to $\operatorname{Ker}(\operatorname{div})^{\perp}=\mathcal{R}$ (grad). This proves the decomposition (B.8). 
Corollary B.3. Let $\boldsymbol{f} \in \boldsymbol{L}^{p}\left(\Omega_{e}\right), 1 \leq p<2$, such that $\operatorname{curl} \boldsymbol{f} \in L^{2}(\Omega)$ and $\gamma_{t} \boldsymbol{f}=0$ on $\Gamma_{\infty}$. Then $\boldsymbol{f}$ admits the following decomposition,

$$
f=\boldsymbol{f}_{r}+\nabla \varphi
$$

with $\boldsymbol{f}_{r} \in H^{1}\left(\Omega_{e}\right), \gamma_{t} \boldsymbol{f}_{r}=0$ on $\Gamma_{\infty}$, and $\varphi \in W_{0, \Gamma_{\infty}}^{1, p}\left(\Omega_{e}\right)$ such that

$$
\left\|\boldsymbol{f}_{r}\right\|_{1, \Omega_{e}} \lesssim\|\operatorname{curl} \boldsymbol{f}\|_{0, \Omega_{e}} \text {. }
$$

Proof. Consider the unique variational solution $\psi \in H_{0, \Gamma_{0}}^{1}\left(\Omega_{e}\right)$ of the mixed boundary value problem

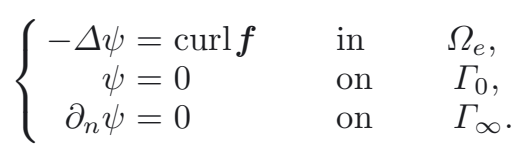

Now, notice that $\Omega_{e}$ is regular except at $\boldsymbol{x}_{0}$. According to well-known results for the Laplace operator in polygonal domains with reentrant corners ( $c f$. for example [26]), $\psi$ admits the following decomposition

$$
\psi=\psi_{r}+c \eta_{0} r_{0}^{\lambda} \sin \left(\lambda \theta_{0}\right)
$$

with $\psi_{r} \in H^{2}\left(\Omega_{e}\right) \cap H_{0, \Gamma_{0}}^{1}\left(\Omega_{e}\right)$ and $\partial_{n} \psi_{r}=0$ on $\Gamma_{\infty}$. In the above decomposition, $\left(r_{0}, \theta_{0}\right)$ are the local polar coordinates with respect to $\boldsymbol{x}_{0}$ and $\eta_{0}$ is a radial cut-off function with respect to $\boldsymbol{x}_{0} . \lambda=\pi / \omega_{e}$ is the first singular exponent of the Laplace operator with Dirichlet boundary condition in a sector with opening angle $\omega_{e}$ at $\boldsymbol{x}_{0}$. Notice also that the singular coefficient $c$ vanishes if $\omega_{e} \leq \pi$. We further have the a priori-estimate

$$
\left\|\psi_{r}\right\|_{2, \Omega_{e}}+|c| \lesssim\|\operatorname{curl} \boldsymbol{f}\|_{0, \Omega_{e}}
$$

Now, according to Theorem B.2, the field $\boldsymbol{f}_{0}=\boldsymbol{f}-\boldsymbol{c u r l} \psi$ is a gradient and $\boldsymbol{f}$ thus reads

$$
\boldsymbol{f}=\operatorname{curl}\left(\psi_{r}+c \eta_{0} r_{0}^{\lambda} \sin \left(\lambda \theta_{0}\right)\right)+\nabla \varphi_{0}
$$

with $\varphi_{0} \in W_{0, \Gamma_{\infty}}^{1, p}\left(\Omega_{e}\right)$. A simple computation shows that

$$
\operatorname{curl}\left(\eta_{0} r_{0}^{\lambda} \sin \left(\lambda \theta_{0}\right)\right)=\nabla\left(\eta_{0} r_{0}^{\lambda} \cos \left(\lambda \theta_{0}\right)\right)+\boldsymbol{g}_{r} .
$$

Here, $\boldsymbol{g}_{r}$ is a regular field depending only on $\eta_{0}^{\prime}$ and the singular coefficient $\lambda$ :

$$
\boldsymbol{g}_{r}\left(r_{0}, \theta_{0}\right)=\eta_{0}^{\prime}\left(r_{0}\right) r_{0}^{\lambda} \sin \left(\lambda \theta_{0}\right)\left(\begin{array}{c}
\cos \theta_{0}+\sin \theta_{0} \\
\sin \theta_{0}-\cos \theta_{0}
\end{array}\right) .
$$

Now, the function $S^{\mathrm{Neu}, \omega_{e}}\left(r_{0}, \theta_{0}\right)=r_{0}^{\lambda} \cos \left(\lambda \theta_{0}\right)$ which is actually the singular function for a Neumann problem in a sector with angle $\omega_{e}>\pi$, belongs to $H^{1}\left(\Omega_{e}\right)$ and thus to $W^{1, p}\left(\Omega_{e}\right)$ for $1 \leq p<2$. This proves (B.9) with $\boldsymbol{f}_{r}=\operatorname{curl} \psi_{r}+c \boldsymbol{g}_{r}$ and $\varphi=\varphi_{0}+c \eta_{0} S^{\mathrm{Neu}, \omega_{e}}$. We clearly have $\gamma_{t} \boldsymbol{f}_{r}=\partial_{n} \psi_{r}+c \gamma_{t} \boldsymbol{g}_{r}=0$ on $\Gamma_{\infty}$. Estimate (B.10) follows from (B.11).

\section{REFERENCES}

[1] A. Alonso Rodríguez and A. Valli, Eddy Current Approximation of Maxwell Equations, Springer, Milan (2010).

[2] R. Albanese and G. Rubinacci, Magnetostatic field computations in terms of two component vector potentials. Int. J. Numer. Methods Engrg. 29 (1990) 515-532.

[3] H. Ammari, A. Buffa and J.-C. Nédélec, A justification of eddy currents model for the Maxwell equations. SIAM J. Appl. Math. 60 (2000) 1805-1823.

[4] C. Amrouche, C. Bernardi, M. Dauge and V. Girault, Vector potentials in three-dimensional non-smooth domains. Math. Methods Appl. Sci. 21 (1998) 823-864.

[5] C. Amrouche and N. El Houda Seloula, $L^{p}$-theory for vector potentials and Sobolev's inequalities for vector vields. C.R. Acad. Sci. Paris Ser. I 349 (2011) 529-534. 
[6] F. Assous, P. Ciarlet, Jr. and E. Sonnendrücker, Resolution of the Maxwell equations in a domain with reentrant corners. Math. Model. Numer. Anal. 32 (1998) 359-389.

[7] A. Bermúdez, R. Rodríguez and P. Salgado, Numerical analysis of electric field formulations of the eddy current model. Numer. Math. 102 (2005) 181-201.

[8] O. Bíró, Edge element formulations of eddy current problems. Comput. Methods Appl. Mech. Engrg. 169 (1999) $391-405$.

[9] O. Bíró and A. Valli, The Coulomb gauged vector potential formulation for the eddy-current problem in general geometry: Well-posedness and numerical approximation. Comput. Methods Appl. Mech. Engrg. 196 (2007) 1890-1904.

[10] A.-S. Bonnet-Ben Dhia, C. Hazard and S. Lohrengel, A singular field method for the solution of Maxwell's equations in polyhedral domains. SIAM J. Appl. Math. 56 (1999) 2028-2044.

[11] J.R. Bowler, Eddy-current interaction with an ideal crack. I. The forward problem. J. Appl. Phys. 75 (1994) $8128-8137$.

[12] J.R. Bowler, S.J. Norton and D.J. Harrison, Eddy-current interaction with an ideal crack. II. The inverse problem. J. Appl. Phys. 75 (1994) 8138-8144.

[13] J.R. Bowler, Theory of eddy current crack response, Technical Report. Iowa State University, Center for Nondestructive Evaluation, Ames IA (2002).

[14] J.R. Bowler, Thin-skin eddy-current inversion for the determination of crack shapes. Inverse Probl. 18 (2002) $1891-1905$.

[15] J.R. Bowler, Y. Yoshida and N. Harfield, Vector-Potential Boundary-Integral Evaluation of Eddy-Current Interaction with a Crack. IEEE Trans. Magn. 33 (1997) 4287-4294.

[16] A. Buffa and P. Ciarlet, Jr., On traces for functional spaces related to Maxwell equations, Part I: An integration by parts formula in Lipschitz polyhedra. Math. Methods Appl. Sci. 24 (2001) 9-30.

[17] M. Costabel and M. Dauge, Singularities of electromagnetic fields in polyhedral domains. Arch. Ration. Mech. Anal. 151 (2000) 221-276.

[18] M. Costabel and M. Dauge, Crack singularities for general elliptic systems. Math. Nachr. 235 (2002), pp. 29-49.

[19] M. Costabel and M. Dauge, Asymptotics without logarithmic terms for crack problems, Commun. Partial. Differ. Eq. 28 (2003) 869-926.

[20] M. Costabel, M. Dauge and S. Nicaise, Singularities of eddy current problems. ESAIM: M2AN 37 (2003) 807-831.

[21] E. Creusé, S. Nicaise, Z. Tang, Y. Le Menach, N. Nemitz and F. Piriou, Residual-based a posteriori estimators for the $\boldsymbol{A}-\boldsymbol{\varphi}$ magnetodynamic harmonic formulation of the Maxwell system. Math. Models Methods Appl. Sci. 22 (2012) DOI : $10.1142 /$ S021820251150028X.

[22] R.W. Freund, Conjugate gradient-type methods for linear systems with complex symmetric coefficient matrices. SIAM J. Sci. Statis. Comput. 13 (1992) 425-448.

[23] T.-P. Fries and M. Baydoun, Crack propagation with the extended finite element method and a hybrid explicit-implicit crack description. Int. J. Numer. Methods Engrg. 89 (2012) 1527-1558.

[24] V. Girault and P.-A. Raviart, Finite Element Methods for Navier-Stokes Equations, Springer, Berlin (1986).

[25] P. Grisvard, Elliptic Problems in Nonsmooth Domains. Pitman, London (1985).

[26] P. Grisvard, Singularities in boundary value problems. Masson, Paris (1992).

[27] N. Harfield and J.R. Bowler, Analysis of eddy-current interaction with a surface-breaking crack. J. Appl. Phys. 76 (1994) 4853-4856.

[28] C. Hazard and M. Lenoir, On the solution of time-harmonic scattering problems for Maxwell's equations. SIAM J. Math. Anal. 27 (1996) 1597-1630.

[29] R. Hiptmair, Symmetric coupling for eddy current problems. SIAM J. Numer. Anal. 40 (2002) 41-65.

[30] M. Křižek and P. Neittaanmäki, Finite element approximation for a div-rot system with mixed boundary conditions in nonsmooth domains. Apl. Mat. 29 (1984) 272-285.

[31] F. Lefèvre, S. Lohrengel and S. Nicaise, An eXtended Finite Element Method for 2D edge elements. Int. J. Numer. Anal. Model. 8 (2011) 641-666.

[32] N. Moës, J. Dolbow and T. Belytschko, A finite element method for crack growth without remeshing. Int. J. Numer. Methods Engrg. 46 (1999) 131-150.

[33] P. Monk, Finite Element Methods for Maxwell's Equations. Oxford University Press (2003).

[34] P.A. Pinsky, Partial Differential Equations and Boundary-Value Problems with Applications. McGraw-Hill, Singapore (1998).

[35] K. Preis, I. Bardi, O. Bíró, C. Magele, G. Vrisk and K.R. Richter, Different finite element formulations of 3D Magnetostatics fields. IEEE Trans. Magn. 28 (1992) 1056-1059.

[36] Z. Ren, Influence of the R.H.S. on the Convergence Behaviour of the Curl-Curl Equation. IEEE Trans. Magn. 32 (1996) $655-658$.

[37] N. Sukumar, D.L. Chopp and B. Moran, Extended finite element method and fast marching method for three-dimensional fatigue crack propagation. Engrg. Fracture Mech. 70 (2003) 29-48. 